\title{
Correlations of eigenvalues and Riemann zeros
}

\author{
John Brian Conrey and Nina Claire Snatth
}

\begin{abstract}
Interest in comparing the statistics of the zeros of the Riemann zeta function with random matrix theory dates back to the 1970s and the work of Montgomery and Dyson. Twelve years ago Rudnick and Sarnak and, independently, Bogomolny and Keating showed that the $n$-point correlation function of the Riemann zeros, correctly scaled and in the limit of infinite height on the critical line, agrees with the scaling limit of the $n$-correlation of eigenvalues of random unitary matrices. The former piece of work holds only for a restricted class of test functions, and the latter relies on a heuristic method and the conjectures of Hardy and Littlewood. Neither tells us more than the asymptotic limit for the general $n$-correlation. In this article we use the ratios conjecture for average values of the Riemann zeta function to produce the lower order terms in a very precise formula for the $n$-correlation of the Riemann zeros. The same method can be applied rigorously in the random matrix case, yielding a formula which shows identical structure (though with none of the arithmetic details) to the correlations of the Riemann zeros - something which cannot be seen from the classical determinantal formula for the random matrix correlation functions.
\end{abstract}
3.2. Averages of logarithmic derivatives of characteristic polynomials


$\begin{array}{ll}\text { 3.3. Residue identity } & 490\end{array}$

3.4. $n$-Correlation via the Ratios theorem 498

3.5. $n$-Correlation theorem $\quad 500$

4. Correlations of the Riemann zeros 506

4.1. The Ratios conjecture for the zeta-function 506

4.2. Averages of logarithmic derivatives of the Riemann zeta-function $\quad 508$

$\begin{array}{ll}\text { 4.3. Residue identity revisited } & 513\end{array}$

4.4. $n$-Correlation via the Ratios conjecture 519

4.5. $n$-Correlation for the Riemann zeros 522

5. Examples $\quad 523$

$\begin{array}{ll}\text { 5.1. Pair correlation, RMT } & 524\end{array}$

$\begin{array}{ll}\text { 5.2. Pair correlation, } \zeta & 525\end{array}$

5.3. Triple correlation, RMT $\quad 526$

$\begin{array}{ll}\text { 5.4. Triple correlation for } \zeta & 527\end{array}$

$\begin{array}{ll}\text { 5.5. Quadruple correlation, RMT } & \mathbf{5 2 8}\end{array}$

5.6. Quadruple correlation, $\zeta \quad 530$

5.7. Auxiliary functions $\quad 533$

$\begin{array}{lr}\text { References } & 534\end{array}$

\section{Introduction}

In 1972 Montgomery [17] and Dyson [12] discovered that pairs of zeros of the Riemann zeta-function are distributed like pairs of eigenvalues of random 
unitary matrices. Part of this discovery could be proven, under the assumption of the Riemann hypothesis, and part relies on a heuristic based on the Hardy-littlewood conjectures for the distribution of prime pairs.

Odlyzko [18], in the 1980s carried out a substantial numerical test of Montgomery's conjecture which provided stunning visual substantiation.

Subsequently Rudnick and Sarnak [20] showed that the limit high on the critical line of the $n$-correlation of the zeros of the Riemann zeta-function agreed with that of unitary matrices, provided that the test function had a Fourier transform with limited support.

At the same time, Bogomolny and Keating [2] showed how the HardyLittlewood conjectures could be used to derive the asymptotic limit of the $n$-correlation.

Bogomolny and Keating [1] also investigated the difference between Montgomery's limiting pair-correlation conjecture and the data of Odlyzko. A close examination of Odlyzko's data revealed that lower-order terms, likely to be of an arithmetic nature, were present. They derived formulae for these lower-order terms, initially using the Hardy-Littlewood conjectures, but subsequently developing a method whose point of departure was the trace formula of Gutzwiller. They gave full details for the lower-order terms for the 2-point correlation, as well as numerics showing the goodness of fit, whereas for three and higher correlations, they outlined several methods which lead to these lower-order terms.

In this paper, we present a different approach to obtaining these lowerorder terms for $n$-correlation. Our approach is based on the 'Ratios conjecture' of Conrey, Farmer and Zirnbauer [6,7] (see also [10]). Assuming the Ratios conjecture we prove a formula which explicitly gives all of the lower-order terms in any order correlation. (In the final section we write down the first four correlations.)

Our method works equally well for the random matrix theory. An interesting feature of this work is the new formula for the $n$-correlation of the random matrix theory that arises by this method (see Theorem 3.5). It is a far less elegant formula than the usual determinantal expression, but it allows for direct comparison with the number theoretical result, illustrating the identical structure of the $n$-point correlations of Riemann zeros and random matrix eigenvalues. (See [15, Remark 1.8] for another example where a formula more complicated than the determinantal one actually yields an easier comparison with the number theory.) In fact, in the scaling limit (when the variables in the test function are multiplied by $\log T / 2 \pi$ and $T$, the height up the critical line, becomes large) all of the arithmetic features of the formula for the $n$-correlation of the Riemann zeros disappear and 
it exactly matches our new formula for the $n$-correlation of eigenvalues of unitary matrices in the equivalent limit. This identification allows us to prove that in the scaling limit the leading order terms for our $n$-correlation of the Riemann zeros have the expected determinantal form. See [9] for the explicit derivation of the asymptotic limit in the case of the triple correlation of Riemann zeros. The higher correlations follow in exactly the same way.

This point is significant in view of the difficulty in making this identification in other works on $n$-correlation and $n$-level density. In Rudnick and Sarnak [20] this identification is proven in the case of test functions restricted to $[-1,1]$; the proof is quite involved and makes serious use of the restriction on the support of the test function. Indeed this point forms a difficulty which shows up, for example, in the work of Gao [14] on $n$-level density for zeros of quadratic $L$-functions. Rubinstein [19] had evaluated this for test functions whose total support was contained in $[-1,1]$ and verified the determinantal form for functions restricted to this class, analogous to what Rudnick and Sarnak did. Gao extended the range of support to $[-2,2]$, but for these test functions he was unable to derive the determinantal form, due to combinatorial complexities. Here we handle the case in full generality without any mention of the test function. It is possible that our method sheds light on this difficulty that arises in these other works.

This paper extends the calculation of the triple correlation of Riemann zeros [9]. An anticipated application of this current work is the determination of the lower-order terms in the nearest neighbor spacing for zeta-zeros.

Throughout this paper we assume the truth of the Riemann Hypothesis.

\section{Background and notation}

\subsection{The Riemann zeta-function}

For more details about the Riemann zeta-function, see the standard texts $[13,21]$, but most of the properties that are needed for this paper are discussed below. The Riemann zeta-function is defined by

$$
\zeta(s)=\sum_{n=1}^{\infty} \frac{1}{n^{s}}
$$

for $s=\sigma+i t$ with $\sigma>1$. It has a meromorphic continuation to the whole complex plane with its only singularity a simple pole at $s=1$ with residue 1 . 
It satisfies a functional equation which, in its symmetric form reads

$$
\pi^{-s / 2} \Gamma\left(\frac{s}{2}\right) \zeta(s)=\pi^{(s-1) / 2} \Gamma\left(\frac{1-s}{2}\right) \zeta(1-s),
$$

and in its asymmetric form is

$$
\zeta(s)=\chi(s) \zeta(1-s)
$$

where

$$
\chi(1-s)=\chi(s)^{-1}=2(2 \pi)^{-s} \Gamma(s) \cos \frac{\pi s}{2} .
$$

The product formula discovered by Euler is

$$
\zeta(s)=\prod_{p}\left(1-\frac{1}{p^{s}}\right)^{-1}
$$

for $\sigma>1$ where the product is over the prime numbers $p$.

The complex zeros of the Riemann zeta-function are denoted by $\rho=\beta+i \gamma_{j}$, where it is known that $0<\beta<1$. The Riemann hypothesis asserts that $\beta=1 / 2$ for all zeros $\rho$. We assume this is true and denote the zeros as $1 / 2+i \gamma_{j}$, where $0<\gamma_{1} \leq \gamma_{2} \leq \cdots$. The number of $\gamma$ with $0<\gamma \leq T$ is given by

$$
N(T)=\#\{\gamma \leq T\}=\frac{T}{2 \pi} \log \frac{T}{2 \pi e}+\mathrm{O}(\log T)
$$

so that the average distance from one $\gamma$ to the next is $\sim 2 \pi / \log \gamma$.

The family $\{\zeta(1 / 2+i t) \mid t>0\}$ parameterized by real numbers $t$ can be modeled by characteristic polynomials of unitary matrices.

\subsection{Unitary matrices}

If $X$ is an $N \times N$ matrix with complex entries $X=\left(x_{j k}\right)$, we let $X^{*}$ be its conjugate transpose, i.e., $X^{*}=\left(y_{j k}\right)$, where $y_{j k}=\overline{x_{k j}} . X$ is said to be unitary if $X X^{*}=I$. We let $U(N)$ denote the group of all $N \times N$ unitary matrices. This is a compact Lie group and has a Haar measure which allows us to analyse. 
All of the eigenvalues of $X \in U(N)$ have absolute value 1; we write them as

$$
e^{i \theta_{1}}, e^{i \theta_{2}}, \ldots, e^{i \theta_{N}}
$$

The eigenvalues of $X^{*}$ are $e^{-i \theta_{1}}, \ldots, e^{-i \theta_{N}}$. Clearly, the determinant, $\operatorname{det} X=$ $\prod_{n=1}^{N} e^{i \theta_{n}}$, of a unitary matrix is a complex number with absolute value equal to 1 .

The average distance from one $\theta$ to the next is $2 \pi / N$. To obtain a sequence of numbers with average spacing 1 we let

$$
\tilde{\theta}_{j}=\frac{N \theta_{j}}{2 \pi}
$$

For any sequence of $N$ points on the unit circle there are matrices in $U(N)$ with these points as eigenvalues. The collection of all matrices with the same set of eigenvalues constitutes a conjugacy class in $U(N)$. Thus, the set of conjugacy classes can be identified with the collection of sequences of $N$ points on the unit circle.

We are interested in computing various statistics about these eigenvalues. Consequently, we identify all matrices in $U(N)$ that have the same set of eigenvalues. Weyl's integration formula gives a simple way to perform averages over $U(N)$ for functions $f$ that are constant on conjugacy classes. Such functions are called "class functions". Weyl's formula asserts that for such an $f$,

$$
\int_{U(N)} f(X) d \text { Haar }=\int_{[0,2 \pi]^{N}} f\left(\theta_{1}, \ldots, \theta_{N}\right) d X_{N}
$$

where

$$
d X_{N}=\prod_{1 \leq j<k \leq N}\left|e^{i \theta_{k}}-e^{i \theta_{j}}\right|^{2} \frac{d \theta_{1} \cdots d \theta_{N}}{N !(2 \pi)^{N}}
$$

Since $N$ will be fixed in this paper, we will usually write $d X$ in place of $d X_{N}$. The Haar measure can be expressed in terms of the Vandermonde determinant

$$
\Delta\left(w_{1}, \ldots, w_{R}\right)=\operatorname{det}_{R \times R}\left(w_{i}^{j-1}\right)=\prod_{1 \leq j<k \leq R}\left(w_{k}-w_{j}\right)
$$


The characteristic polynomial of a matrix $X$ is denoted $\Lambda_{X}(s)$ and is defined by

$$
\Lambda_{X}(s)=\operatorname{det}\left(I-s X^{*}\right)=\prod_{n=1}^{N}\left(1-s e^{-i \theta_{n}}\right) .
$$

The roots of $\Lambda_{X}(s)$ are the eigenvalues of $X$ and are on the unit circle. The characteristic polynomial satisfies the functional equation

$$
\begin{aligned}
\Lambda_{X}(s) & =(-s)^{N} \prod_{n=1}^{N} e^{-i \theta_{n}} \prod_{n=1}^{N}\left(1-e^{i \theta_{n}} / s\right) \\
& =(-1)^{N} \operatorname{det} X^{*} s^{N} \Lambda_{X^{*}}(1 / s) .
\end{aligned}
$$

Note that

$$
s \frac{\Lambda_{X}^{\prime}}{\Lambda_{X}}(s)+\frac{1}{s} \frac{\Lambda_{X^{*}}^{\prime}}{\Lambda_{X^{*}}}\left(\frac{1}{s}\right)=N .
$$

These characteristic polynomials have value distributions similar to those of the Riemann zeta-function and form the basis of random matrix models which predict behavior for the Riemann zeta-function based on what can be proven about the $\Lambda$. Some care has to be taken in making these comparisons because we are used to thinking about the zeta-function in a half-plane, whereas the $\Lambda$ are naturally studied in a circle. The translation is that the $1 / 2$-line corresponds to the unit circle; the half-plane to the right of the $1 / 2$-line corresponds to the inside of the unit circle. Note that $\Lambda_{X}(0)=1$ is the analog of $\lim _{\sigma \rightarrow \infty} \zeta(\sigma+i t)=1$.

We let

$$
z(x)=\frac{1}{1-e^{-x}} .
$$

In our formulas for averages of characteristic polynomials the function $z(x)$ plays the role for random matrix theory that $\zeta(1+x)$ plays in the theory of moments of the Riemann zeta-function.

We want an accurate formula for

$$
\sum_{0<\gamma_{j_{1}}, \ldots, \gamma_{j_{n}}<T}^{*} f\left(\gamma_{j_{1}}, \ldots, \gamma_{j_{n}}\right),
$$

for suitable functions $f$, to be described later, where the sum is for distinct indices $j$; the desired formula should be analogous to the RMT theorem which we state in the next section. 


\section{Eigenvalue correlations}

Here is a statement for $n$-correlation of eigenvalues of random unitary matrices of size $N$ :

Theorem 3.1. Let $f:[0,2 \pi]^{n} \rightarrow \mathbb{C}$ be a continuous function of $n$-variables. Then

$$
\begin{aligned}
& \int_{U(N))_{1 \leq j_{1}, \ldots, j_{n} \leq N}} \sum^{*} f\left(\theta_{j_{1}}, \ldots, \theta_{j_{n}}\right) d X_{N} \\
& \quad=\frac{1}{(2 \pi)^{n}} \int_{[0,2 \pi]^{n}} f\left(\theta_{1}, \ldots, \theta_{n}\right) \operatorname{det}_{n \times n} S_{N}\left(\theta_{k}-\theta_{j}\right) d \theta_{1} \cdots d \theta_{n}
\end{aligned}
$$

where $\sum^{*}$ indicates that the sum is for distinct indices and where

$$
S_{N}(\theta)=\frac{\sin (N \theta / 2)}{\sin (\theta / 2)}
$$

This theorem is a well-known consequence of Gaudin's lemma; see [4].

In the following sections we present a new proof of this theorem, for periodic, holomorphic test functions $f$, based on a formula for averaging ratios of characteristic polynomials of unitary matrices. There are many proofs for this formula; see $[3,7,8]$. The point of this approach is that it has a natural analog in the theory of $L$-functions.

\subsection{Averages of ratios of characteristic polynomials}

The statement of the Ratios theorem is slightly complicated. We attempt to make it easier to comprehend by eliminating subscripts. So, let there be given finite sets $A, B, C$ and $D$ and consider

$$
\mathcal{R}(A, B ; C, D):=\int_{U(N)} \frac{\prod_{\alpha \in A} \Lambda_{X}\left(e^{-\alpha}\right) \prod_{\beta \in B} \Lambda_{X^{*}}\left(e^{-\beta}\right)}{\prod_{\gamma \in C} \Lambda_{X}\left(e^{-\gamma}\right) \prod_{\delta \in D} \Lambda_{X^{*}}\left(e^{-\delta}\right)} d X
$$

with $\Re \gamma>0, \Re \delta>0$. Theorem 3.2, the Ratios theorem, is written in an equivalent but slightly different form to previous work, where we express 
$\mathcal{R}(A, B ; C, D)$ as a sum over subsets $S \subset A$ and $T \subset B$ with $|S|=|T|$. Each term in this sum essentially has the same structure, except that the elements of $S$ effectively exchange places with those in $T$. In addition we let $\bar{S}=$ $A-S$ and $\bar{T}=B-T$. We will let $\hat{\alpha}$ denote a generic member of $S$ and $\hat{\beta}$ denote a generic member of $T$; we will use $\alpha$ and $\beta$ for generic members of $A$ and $B$ or of $\bar{S}$ and $\bar{T}$, according to the context. Also $S^{-}=\{-\hat{\alpha}$ : $\hat{\alpha} \in S\}$, and similarly for $T^{-}$. The Ratios theorem is most easily stated in terms of

$$
Z(A, B):=\prod_{\substack{\alpha \in A \\ \beta \in B}} z(\alpha+\beta)
$$

where $z(x)=1 /\left(1-e^{-x}\right)$, and

$$
\begin{aligned}
& Z(A, B ; C, D):=\frac{\prod_{\substack{\alpha \in A \\
\beta \in B}} z(\alpha+\beta) \prod_{\substack{\gamma \in C \\
\delta \in D}} z(\gamma+\delta)}{\prod_{\substack{\alpha \in A \\
\delta \in D}} z(\alpha+\delta) \prod_{\substack{\beta \in B \\
\gamma \in C}} z(\beta+\gamma)} \\
& =\frac{Z(A, B) Z(C, D)}{Z(A, D) Z(B, C)} .
\end{aligned}
$$

Theorem 3.2 (Ratios Theorem [7,8]). With $\Re \gamma>0, \Re \delta>0$ for $\gamma \in C$ and $\delta \in D,|C| \leq|A|+N$ and $|D| \leq|B|+N$, we have

$$
\mathcal{R}(A, B ; C, D)=\sum_{\substack{S \subset A, T \subset B \\|S|=|T|}} e^{-N\left(\sum_{\hat{\alpha} \in S} \hat{\alpha}+\sum_{\hat{\beta} \in T} \hat{\beta}\right)} Z\left(\bar{S}+T^{-}, \bar{T}+S^{-} ; C, D\right)
$$

where $A=S+\bar{S}, B=T+\bar{T}$ and $Z$ is defined in (3.3).

\subsection{Averages of logarithmic derivatives of characteristic polynomials}

For use in determining multiple correlation we differentiate the Ratios theorem to obtain a theorem about averages of logarithmic derivatives: 
Theorem 3.3. If $\Re \alpha_{j}>0$ and $\Re \beta_{j}>0$ for $\alpha_{j} \in A$ and $\beta_{j} \in B$, then $J(A ; B)$ $=J^{*}(A ; B)$ where

$$
\begin{aligned}
J(A ; B) & :=\int_{U(N)} \prod_{\alpha \in A}\left(-e^{-\alpha}\right) \frac{\Lambda_{X}^{\prime}}{\Lambda_{X}}\left(e^{-\alpha}\right) \prod_{\beta \in B}\left(-e^{-\beta}\right) \frac{\Lambda_{X^{*}}^{\prime}}{\Lambda_{X^{*}}}\left(e^{-\beta}\right) d X, \\
J^{*}(A ; B) & :=\sum_{\substack{S \subset A, T \subset B \\
|S|=|T|}} e^{-N\left(\sum_{\hat{\alpha} \in S} \hat{\alpha}+\sum_{\hat{\beta} \in T} \hat{\beta}\right)} \frac{Z(S, T) Z\left(S^{-}, T^{-}\right)}{Z^{\dagger}\left(S, S^{-}\right) Z^{\dagger}\left(T, T^{-}\right)}
\end{aligned}
$$

$$
\times \sum_{\substack{(A-S)+(B-T) \\=U_{1}+\cdots+U_{R} \\\left|U_{r}\right| \leq 2}} \prod_{r=1}^{R} H_{S, T}\left(U_{r}\right),
$$

and

$$
H_{S, T}(W)= \begin{cases}\sum_{\hat{\alpha} \in S} \frac{z^{\prime}}{z}(\alpha-\hat{\alpha})-\sum_{\hat{\beta} \in T} \frac{z^{\prime}}{z}(\alpha+\hat{\beta}) & \text { if } W=\{\alpha\} \subset A-S, \\
\sum_{\hat{\beta} \in T} \frac{z^{\prime}}{z}(\beta-\hat{\beta})-\sum_{\hat{\alpha} \in S} \frac{z^{\prime}}{z}(\beta+\hat{\alpha}) & \text { if } W=\{\beta\} \subset B-T, \\
\left(\frac{z^{\prime}}{z}\right)^{\prime}(\alpha+\beta) & \text { if } W=\{\alpha, \beta\} \text { with } \\
0 & \begin{array}{c}
\alpha \in A-S, \beta \in B-T \\
\text { otherwise. }
\end{array}\end{cases}
$$

Also, $Z(A, B)=\prod_{\substack{\alpha \in A \\ \beta \in B}} z(\alpha+\beta)$, with the dagger on $Z^{\dagger}\left(S, S^{-}\right)$imposing the additional restriction that a factor $z(x)$ is omitted if its argument is zero.

Remark 3.1. The definitions of $J(A ; B)$ and $J^{*}(A ; B)$ make sense without the restriction that $\Re \alpha_{j}>0$ and $\Re \beta_{j}>0$. However, the two are not equal without these restrictions.

Remark 3.2. Note that $J^{*}(A ; B)$ has a pole when an $\alpha \in A$ is equal to $-\beta$, for some $\beta \in B$. It also appears to have a pole when two $\alpha$ s are equal, say $\alpha_{1}=\alpha_{2}$, occurring when $\alpha_{1} \in S$ and $\alpha_{2} \notin S$, as seen in the term $\frac{z^{\prime}}{z}(\alpha-\hat{\alpha})$ of (3.21). However, this is cancelled by a pole with residue of the opposite 
sign when $S$ is replaced by $S-\left\{\alpha_{1}\right\}+\left\{\alpha_{2}\right\}$. The same phenomenon occurs when two $\beta \mathrm{s}$ are equal, as can be seen from the concrete examples given in (5.23) and (5.29).

Proof of Theorem 3.3. By (3.1), we have

$$
J(A ; B)=\left.\prod_{\substack{\alpha \in A \\ \beta \in B}} \frac{d}{d \alpha} \frac{d}{d \beta} \mathcal{R}(A, B ; C, D)\right|_{\substack{C=A \\ D=B}} .
$$

Of course, in this situation $|C|=|A|$ and $|D|=|B|$; so that we may think of $A=\left\{\alpha_{1}, \ldots, \alpha_{k}\right\}$ and $C=\left\{\gamma_{1}, \ldots, \gamma_{k}\right\}$ and then the substitution " $C=A$ " means the substitution $\gamma_{i}=\alpha_{i}$ for $i=1,2, \ldots, k$, and similarly for $D$ and $B$.

Recall from Theorem 3.2 that $\mathcal{R}$ is expressed as a sum of $Z$ over subsets $S$ and $T$. In performing the differentiations in (3.7) we will find that the derivatives with respect to the variables in $S$ and $T$ are fairly simple to perform (as we will show below, culminating in (3.14)), but we will need Lemma 3.1 to differentiate with respect to the remaining variables. Hence we first rewrite $Z$ so as to separate these variable types.

Note first that $Z(A, B)=Z(B, A)$ and that $Z$ behaves nicely with respect to unions:

$$
Z\left(A_{1}+A_{2}, B\right)=Z\left(A_{1}, B\right) Z\left(A_{2}, B\right) .
$$

Recall that $A=S+\bar{S}$ and $B=T+\bar{T}$ and put $C=C_{S}+C_{\bar{S}}$ and $D=$ $D_{T}+D_{\bar{T}}$, where we think of $C_{S}$, for example, as being the set that will be substituted by $S$ when eventually $C$ is substituted by $A$. Then using (3.8) repeatedly, we have

$$
\begin{aligned}
& Z\left(\bar{S}+T^{-}, \bar{T}+S^{-} ; C, D\right) \\
& \quad=\frac{Z(\bar{S}, \bar{T}) Z\left(\bar{S}, S^{-}\right) Z\left(T^{-}, \bar{T}\right) Z\left(T^{-}, S^{-}\right) Z(C, D)}{Z(\bar{S}, D) Z(\bar{T}, C) Z\left(S^{-}, C_{\bar{S}}\right) Z\left(T^{-}, D_{\bar{T}}\right) Z\left(S^{-}, C_{S}\right) Z\left(T^{-}, D_{T}\right)} .
\end{aligned}
$$

This simplifies further if we make the substitution for $C$ and $D$ :

$$
\begin{aligned}
\left.Z\left(\bar{S}+T^{-}, \bar{T}+S^{-} ; C, D\right)\right|_{\substack{C=A \\
D=B}} & =\frac{Z(S, T) Z\left(S^{-}, T^{-}\right)}{Z\left(S, S^{-}\right) Z\left(T, T^{-}\right)} \\
& =Z\left(S, T ; T^{-}, S^{-}\right) .
\end{aligned}
$$


Note that since $z(x)$ has a pole at $x=0$, the resulting expression is 0 unless both $S$ and $T$ are empty.

We now differentiate (3.9) with respect to the variables in $S$ and $T$; these derivatives are easy to calculate because, anticipating the substitution of each $\gamma \in C_{S}$ by an $\hat{\alpha}$ we see that in differentiating with respect to $\hat{\alpha}$ the expression $z(\gamma-\hat{\alpha})$ in the denominator (one of the factors of $Z\left(S^{-}, C_{S}\right)$ ) must be differentiated; if not it makes the whole expression 0 after the substitution is made because $z(x)$ has a pole at $x=0$. Using the notation

$$
Z^{\dagger}(X, Y)=\prod_{\substack{x \in X, y \in Y \\ x+y \neq 0}} z(x+y)
$$

and noting that

$$
\frac{d}{d \hat{\alpha}} \frac{1}{z(\gamma-\hat{\alpha})}=-e^{\hat{\alpha}-\gamma}
$$

we have, for example,

$$
\left.\prod_{\hat{\alpha} \in S} \frac{d}{d \hat{\alpha}} \frac{1}{Z\left(S^{-}, C_{S}\right)}\right|_{C_{S}=S}=\frac{(-1)^{|S|}}{Z^{\dagger}\left(S^{-}, S\right)}
$$

In this way we obtain

$$
\begin{aligned}
J(A ; B)= & \sum_{\substack{S, T \\
|S|=|T|}} e^{-N\left(\sum \hat{\alpha}+\sum \hat{\beta}\right)} \frac{Z(S, T) Z\left(S^{-}, T^{-}\right)}{Z^{\dagger}\left(S, S^{-}\right) Z^{\dagger}\left(T, T^{-}\right)} \\
& \times \prod_{\substack{\alpha \in \bar{S} \\
\beta \in \bar{T}}} \frac{d}{d \alpha} \frac{d}{d \beta} \\
& \times\left.\left(\frac{Z(\bar{S}, \bar{T}) Z\left(\bar{S}, S^{-}\right) Z\left(\bar{T}, T^{-}\right) Z(C, D)}{Z(S, T) Z\left(C_{\bar{S}}, S^{-}\right) Z\left(D_{\bar{T}}, T^{-}\right) Z(\bar{S}, D) Z(\bar{T}, C)}\right)\right|_{\substack{C=A \\
D=B}}
\end{aligned}
$$

Note that the sets $C_{\bar{S}}$ and $D_{\bar{T}}$ vary from term to term in the sum over $S$ and $T$ since the division of $C$ into the union of sets $C_{S}$ and $C_{\bar{S}}$ mimics the form of $A=S+\bar{S}$, and similarly for $D$. Also observe that

$$
\left.\frac{Z(\bar{S}, \bar{T}) Z\left(\bar{S}, S^{-}\right) Z\left(\bar{T}, T^{-}\right) Z(C, D)}{Z(S, T) Z\left(C_{\bar{S}}, S^{-}\right) Z\left(D_{\bar{T}}, T^{-}\right) Z(\bar{S}, D) Z(\bar{T}, C)}\right|_{\substack{C=A \\ D=B}}=1 .
$$


To perform the differentiations in (3.14) we use a form of logarithmic differentiation expressed in the following.

Lemma 3.1. Let $H$ be a differentiable function of $w \in W$. Then

$$
\left(\prod_{w \in W} \frac{d}{d w}\right) e^{H}=e^{H} \sum_{W=W_{1}+\cdots+W_{r}} H\left(W_{1}\right) \cdots H\left(W_{r}\right)
$$

where

$$
H(W)=\left(\prod_{w \in W} \frac{d}{d w}\right) H
$$

The sum is over all set partitions of $W$ into disjoint sets $W_{j}$.

In words this Lemma says that to perform a derivative with respect to each variable once, we form all of the set partitions of the complete set of variables and add up over these set partitions the product of the partial derivatives of the exponent $H$ with respect to each variable in each subset of the partition. This lemma is obvious upon working a few examples.

We apply this lemma with

$$
\begin{gathered}
H=H_{S, T}^{A, B, C, D}:=\sum_{\substack{\alpha \in \bar{S} \\
\beta \in \bar{T}}} \log z(\alpha+\beta)+\sum_{\substack{\alpha \in \bar{S} \\
\hat{\alpha} \in S}} \log z(\alpha-\hat{\alpha})+\sum_{\substack{\beta \in \bar{T} \\
\hat{\beta} \in T}} \log z(\beta-\hat{\beta}) \\
\text { 8) } \quad-\sum_{\substack{\alpha \in \bar{S} \\
\delta \in D}} \log z(\alpha+\delta)-\sum_{\substack{\beta \in \bar{T} \\
\gamma \in C}} \log z(\beta+\gamma)
\end{gathered}
$$

and so obtain, with $H_{S, T}(W):=\left.H_{S, T}^{A, B, C, D}(W)\right|_{\substack{C=A \\ D=B}}=\left(\left(\prod_{w \in W} \frac{d}{d w}\right)\right.$ $\left.H_{S, T}^{A, B, C, D}\right)\left.\right|_{\substack{C=A \\ D=B}}$

$$
J(A ; B)=\sum_{\substack{S, T \\|S|=|T|}} e^{-N\left(\sum \hat{\alpha}+\sum \hat{\beta}\right)} \frac{Z(S, T) Z\left(S^{-}, T^{-}\right)}{Z^{\dagger}\left(S, S^{-}\right) Z^{\dagger}\left(T, T^{-}\right)}
$$

$$
\times \sum_{\substack{\bar{S}+\bar{T} \\=W_{1}+\cdots+W_{R}}} \prod_{r=1}^{R} H_{S, T}\left(W_{r}\right) .
$$


Strictly speaking, $H_{S, T}(W)$ depends on $A$ and $B$, but from now on use of $H_{S, T}(W)$ will refer always to the expressions in (3.21) to (3.26), and these can be used without specifically referring to $A$ and $B$.

By consideration of (3.18) it is clear that we can restrict the subsets $W_{r}$ to be singletons or else pairs which have precisely one $\alpha$ and one $\beta$. This follows from some easy calculations. Since

$$
H_{S, T}^{A, B, C, D}(\{\alpha\})=\sum_{\beta \in \bar{T}} \frac{z^{\prime}}{z}(\alpha+\beta)+\sum_{\hat{\alpha} \in S} \frac{z^{\prime}}{z}(\alpha-\hat{\alpha})-\sum_{\delta \in D} \frac{z^{\prime}}{z}(\alpha+\delta),
$$

we have

$$
H_{S, T}(\{\alpha\})=\sum_{\hat{\alpha} \in S} \frac{z^{\prime}}{z}(\alpha-\hat{\alpha})-\sum_{\hat{\beta} \in T} \frac{z^{\prime}}{z}(\alpha+\hat{\beta}), \quad \alpha \notin S .
$$

Similarly,

$$
H_{S, T}(\{\beta\})=\sum_{\hat{\beta} \in T} \frac{z^{\prime}}{z}(\beta-\hat{\beta})-\sum_{\hat{\alpha} \in S} \frac{z^{\prime}}{z}(\beta+\hat{\alpha}), \quad \beta \notin T .
$$

In addition

$$
H_{S, T}(\{\alpha, \beta\})=\left(\frac{z^{\prime}}{z}\right)^{\prime}(\alpha+\beta), \quad \alpha, \beta \notin S \text { or } T .
$$

Also,

$$
H_{S, T}(\emptyset)=1,
$$

and

$$
H_{S, T}\left(\left\{\alpha, \alpha^{\prime}\right\}\right)=H_{S, T}\left(\left\{\beta, \beta^{\prime}\right\}\right)=0
$$

and

$$
H_{S, T}(W)=0 \quad \text { if }|W| \geq 3 .
$$

\subsection{Residue identity}

A key ingredient of the proof of $n$-correlation will be the following residue identity for $J^{*}(A ; B)$ : 
Lemma 3.2. Suppose that $\alpha^{*} \in A$ and $\beta^{*} \in B$. Let $A^{\prime}=A-\left\{\alpha^{*}\right\}$ and $B^{\prime}=B-\left\{\beta^{*}\right\}$. Then $J^{*}(A ; B)$ has a simple pole at $\alpha^{*}=-\beta^{*}$ with

$$
\underset{\alpha^{*}=-\beta^{*}}{\operatorname{Res}} J^{*}(A ; B)=N J^{*}\left(A^{\prime} ; B^{\prime}\right)+J^{*}\left(A^{\prime} ; B\right)+J^{*}\left(A^{\prime}+\left\{-\beta^{*}\right\} ; B^{\prime}\right) .
$$

Proof. By Theorem 3.3 we have

$$
J^{*}(A, B)=\sum_{\substack{S \subset A \\ T \subset B \\|S|=|T|}} D_{S, T}(\bar{S}, \bar{T}),
$$

where throughout this proof (and this paper) $A=\bar{S}+S, B=\bar{T}+T$ and

$$
D_{S, T}(\bar{S}, \bar{T})=Q(S, T) \sum_{\bar{S}+\bar{T}=\sum W_{r}} \prod_{r} H_{S, T}\left(W_{r}\right) .
$$

Here the sum is over any collection of non-empty sets $W_{1}, W_{2}, \ldots$, which form a partition of $\bar{S}+\bar{T}$,

$$
Q(S, T)=e^{-N\left(\sum_{\hat{\alpha} \in S} \hat{\alpha}+\sum_{\hat{\beta} \in T} \hat{\beta}\right)} \frac{Z(S, T) Z\left(S^{-}, T^{-}\right)}{Z^{\dagger}\left(S, S^{-}\right) Z^{\dagger}\left(T, T^{-}\right)}
$$

and $H_{S, T}(W)$ is defined as in (3.21) to (3.26) and $Z^{\dagger}$ is defined in Theorem 3.3. We claim that $D, Q$ and $H$ have the following properties:

P1: If $\alpha^{*} \in \bar{S}$ and $\beta^{*} \in \bar{T}$, then $Q(S, T)$ is independent of $\alpha^{*}$ and $\beta^{*}$ and

$$
H_{S, T}(W)= \begin{cases}\frac{1}{\left(\alpha^{*}+\beta^{*}\right)^{2}}+\mathrm{O}(1) & \text { if } W=\left\{\alpha^{*}, \beta^{*}\right\} \\ \mathrm{O}(1) & \text { otherwise. }\end{cases}
$$

P2: If $\alpha^{*} \in S$ and $\beta^{*} \in \bar{T}$, then $Q(S, T)$ is regular when $\alpha^{*}=-\beta^{*}$ and

$$
H_{S, T}(W)= \begin{cases}\frac{1}{\alpha^{*}+\beta^{*}}+\mathrm{O}(1) & \text { if } W=\left\{\beta^{*}\right\}, \\ \mathrm{O}(1) & \text { otherwise. }\end{cases}
$$

P3: If $\alpha^{*} \in \bar{S}$ and $\beta^{*} \in T$, then $Q(S, T)$ is regular when $\alpha^{*}=-\beta^{*}$ and

$$
H_{S, T}(W)= \begin{cases}\frac{1}{\alpha^{*}+\beta^{*}}+\mathrm{O}(1) & \text { if } W=\left\{\alpha^{*}\right\} \\ \mathrm{O}(1) & \text { otherwise }\end{cases}
$$


P4: If $\alpha^{*} \in S$ and $\beta^{*} \in T$ and $S^{\prime}=S-\left\{\alpha^{*}\right\}$ and $T^{\prime}=T-\left\{\beta^{*}\right\}$, then $Q(S, T)=\left(-1 /\left(\alpha^{*}+\beta^{*}\right)^{2}+\mathrm{O}(1)\right) Q_{1}(S, T)$, where

$$
\begin{aligned}
Q_{1}(S, T)= & Q\left(S^{\prime}, T^{\prime}\right)\left(1-\left(\alpha^{*}+\beta^{*}\right)\left(N+\left.H_{S^{\prime}, T^{\prime}}\left(\left\{\alpha^{*}\right\}\right)\right|_{\alpha^{*}=-\beta^{*}}\right.\right. \\
& \left.\left.+H_{S^{\prime}, T^{\prime}}\left(\left\{\beta^{*}\right\}\right)\right)+\mathrm{O}\left(\left|\alpha^{*}+\beta^{*}\right|^{2}\right)\right)
\end{aligned}
$$

and

$$
\begin{aligned}
H_{S, T}(W)= & H_{S^{\prime}, T^{\prime}}(W)-\left(\alpha^{*}+\beta^{*}\right) \\
& \times\left(H_{S^{\prime}, T^{\prime}}\left(W+\left\{\alpha^{*}\right\}\right)_{\alpha^{*}=-\beta^{*}}+H_{S^{\prime}, T^{\prime}}\left(W+\left\{\beta^{*}\right\}\right)\right) \\
& +\mathrm{O}\left(\left|\alpha^{*}+\beta^{*}\right|^{2}\right) .
\end{aligned}
$$

We show that the lemma follows from these four properties and then prove that these properties hold in this situation. (We will later demonstrate a proof along very similar lines when we treat the $n$-correlation of the zeta-zeros.)

From these four properties we obtain four Laurent or Taylor expansions of $D_{S, T}(\bar{S}, \bar{T})$ as a function of $\alpha^{*}$ in a neighborhood of $-\beta^{*}$ :

- If $\alpha^{*} \in \bar{S}$ and $\beta^{*} \in \bar{T}$, then (with $\bar{S}^{\prime}=\bar{S}-\left\{\alpha^{*}\right\}$ and $\bar{T}^{\prime}=\bar{T}-\left\{\beta^{*}\right\}$ )

$$
\begin{aligned}
D_{S, T}(\bar{S}, \bar{T}) & =\left(\frac{1}{\left(\alpha^{*}+\beta^{*}\right)^{2}}+\mathrm{O}(1)\right) Q(S, T) \sum_{\bar{S}^{\prime}+\bar{T}^{\prime}=\sum W_{r}} \prod_{r} H_{S, T}\left(W_{r}\right) \\
& =\left(\frac{1}{\left(\alpha^{*}+\beta^{*}\right)^{2}}+\mathrm{O}(1)\right) D_{S, T}\left(\bar{S}^{\prime}, \bar{T}^{\prime}\right)
\end{aligned}
$$

consequently, $\operatorname{Res}_{\alpha^{*}=-\beta^{*}} D_{S, T}(\bar{S}, \bar{T})=0$.

- If $\alpha^{*} \in S$ and $\beta^{*} \in \bar{T}$, then

$$
\begin{aligned}
\underset{\alpha^{*}=-\beta^{*}}{\operatorname{Res}} D_{S, T}(\bar{S}, \bar{T}) & =Q\left(S^{\prime}+\left\{-\beta^{*}\right\}, T\right) \sum_{\bar{S}+\bar{T}^{\prime}=\sum W_{r}} \prod_{r} H_{S^{\prime}+\left\{-\beta^{*}\right\}, T}\left(W_{r}\right) \\
& =D_{S^{\prime}+\left\{-\beta^{*}\right\}, T}\left(\bar{S}, \bar{T}^{\prime}\right) .
\end{aligned}
$$

- If $\alpha^{*} \in \bar{S}$ and $\beta^{*} \in T$, then

$$
\begin{aligned}
\operatorname{Res}_{\alpha^{*}=-\beta^{*}} D_{S, T}(\bar{S}, \bar{T}) & =Q(S, T) \sum_{\bar{S}^{\prime}+\bar{T}=\sum W_{r}} \prod_{r} H_{S, T}\left(W_{r}\right) \\
& =D_{S, T}\left(\bar{S}^{\prime}, \bar{T}\right) .
\end{aligned}
$$


- If $\alpha^{*} \in S$ and $\beta^{*} \in T$, then

$$
\begin{aligned}
Q_{1}(S, T) & \sum_{\bar{S}+\bar{T}=\sum W_{r}} \prod_{r} H_{S, T}\left(W_{r}\right) \\
= & Q\left(S^{\prime}, T^{\prime}\right) \sum_{\bar{S}+\bar{T}=\sum W_{r}} \prod_{r} H_{S^{\prime}, T^{\prime}}\left(W_{r}\right) \\
& \times\left(1+\left(\alpha^{*}+\beta^{*}\right)\left(N+\left.H_{S^{\prime}, T^{\prime}}\left(\left\{\alpha^{*}\right\}\right)\right|_{\alpha^{*}=-\beta^{*}}+H_{S^{\prime}, T^{\prime}}\left(\left\{\beta^{*}\right\}\right)\right.\right. \\
& \left.+\sum_{r} \frac{\left.H_{S^{\prime}, T^{\prime}}\left(W_{r}+\left\{\alpha^{*}\right\}\right)\right|_{\alpha^{*}=-\beta^{*}}+H_{S^{\prime}, T^{\prime}}\left(W_{r}+\left\{\beta^{*}\right\}\right)}{H_{S^{\prime}, T^{\prime}}\left(W_{r}\right)}\right) \\
& \left.+\mathrm{O}\left(\left|\alpha^{*}+\beta^{*}\right|^{2}\right)\right) .
\end{aligned}
$$

Therefore in this final case,

$$
\begin{aligned}
\operatorname{Res}_{\alpha^{*}=-\beta^{*}} D_{S, T}(\bar{S}, \bar{T})= & N D_{S^{\prime}, T^{\prime}}(\bar{S}, \bar{T})+D_{S^{\prime}, T^{\prime}}\left(\bar{S}+\left\{-\beta^{*}\right\}, \bar{T}\right) \\
& +D_{S^{\prime}, T^{\prime}}\left(\bar{S}, \bar{T}+\left\{\beta^{*}\right\}\right) .
\end{aligned}
$$

Note that (3.39) can be written as

$$
D_{S^{\prime}, T^{\prime}}(\bar{S}, \bar{T})\left(1+\mathrm{O}\left(\left|\alpha^{*}+\beta^{*}\right|\right)\right)
$$

By (3.36) and (3.39) the double poles in P1 and P4 cancel because

$$
\sum_{\substack{S \subset A, T \subset B \\|S|=|T| \\\left\{\alpha^{*}\right\} \in S,\left\{\beta^{*}\right\} \in T}} D_{S^{\prime}, T^{\prime}}(\bar{S}, \bar{T})=\sum_{\substack{S \subset A, T \subset B \\|S|=|T| \\\left\{\alpha^{*}\right\} \notin S,\left\{\beta^{*}\right\} \notin T}} D_{S, T}\left(\bar{S}^{\prime}, \bar{T}^{\prime}\right)=J^{*}\left(A^{\prime}, B^{\prime}\right)
$$

therefore, the pole at $\alpha^{*}=-\beta^{*}$ is simple. 
Combining the four bullet-points above, we have (where as usual the primed notation means that $\alpha^{*}$ or $\beta^{*}$ has been removed from that set)

$$
\begin{aligned}
& \operatorname{Res}_{\alpha^{*}=-\beta^{*}} J^{*}(A ; B)=\sum_{\substack{S \subset A, T \subset B \\
\text { s.l. }=T,\left\{\alpha^{*}\right\} \in S,\left\{\beta^{*}\right\} \in T}}\left(N D_{S^{\prime}, T^{\prime}}(\bar{S}, \bar{T})+D_{S^{\prime}, T^{\prime}}\left(\bar{S}+\left\{-\beta^{*}\right\}, \bar{T}\right)\right. \\
& \left.+D_{S^{\prime}, T^{\prime}}\left(\bar{S}, \bar{T}+\left\{\beta^{*}\right\}\right)\right)+\sum_{\substack{S \subset A, T \subset B \\
S I|=| T \mid \\
\left\{\alpha^{*}\right\} \notin S,\left\{\beta^{*}\right\} \in T}} D_{S, T}\left(\bar{S}^{\prime}, \bar{T}\right) \\
& +\sum_{\substack{S \subset A, T \subset B \\
|S||T| \\
\left\{\alpha^{*}\right\} \in S,\left\{\beta^{*}\right\} \notin T}} D_{S^{\prime}+\left\{-\beta^{*}\right\}, T}\left(\bar{S}, \bar{T}^{\prime}\right)
\end{aligned}
$$

Note that since $\alpha^{*}$ does not appear in any of the summands, with the temporary convention that $A^{\prime}=R+\bar{R}$, we can relabel two of the sums as follows:

$$
\begin{aligned}
& \sum_{\substack{S \subset A, T \subset B \\
|S|=|T| \\
\left\{\alpha^{*}\right\} \in S,\left\{\beta^{*}\right\} \in T}} D_{S^{\prime}, T^{\prime}}\left(\bar{S}, \bar{T}+\left\{\beta^{*}\right\}\right)+\sum_{\substack{S \subset A, T \subset B \\
|S|=|T| \\
R \subset A^{\prime}, T \subset B \\
|R|=|T| \\
\left\{\beta^{*}\right\} \notin T}} D_{R, T}(\bar{R}, \bar{T})+\sum_{\substack{\left.\left.R \subset A^{*}\right\} \notin S, T \beta^{*}\right\} \in T \\
|R|,|T| \\
\left\{\beta^{*}\right\} \in T}} D_{R, T}(\bar{R}, \bar{T}) \\
& =J^{*}\left(A^{\prime} ; B\right) .
\end{aligned}
$$

Similarly,

$$
\begin{aligned}
& \left.\left(\sum_{\substack{S \subset A, T \subset B \\
|S||T| \\
\left\{\alpha^{*}\right\} \in S,\left\{\beta^{*}\right\} \in T}} D_{S^{\prime}, T^{\prime}}\left(\bar{S}+\left\{\alpha^{*}\right\}, \bar{T}\right)+\sum_{\substack{S \subset A, T \subset B \\
|S|=|T| \\
\left\{\alpha^{*}\right\} \in S,\left\{\beta^{*}\right\} \notin T}} D_{S, T}\left(\bar{S}, \bar{T}^{\prime}\right)\right)\right|_{\alpha^{*}=-\beta^{*}} \\
& \quad=\left.J^{*}\left(A ; B^{\prime}\right)\right|_{\alpha^{*}=-\beta^{*}}=J^{*}\left(A^{\prime}+\left\{-\beta^{*}\right\} ; B^{\prime}\right) .
\end{aligned}
$$

Thus we have arrived at

$$
\underset{\alpha^{*}=-\beta^{*}}{\operatorname{Res}} J^{*}(A ; B)=N J^{*}\left(A^{\prime} ; B^{\prime}\right)+J^{*}\left(A^{\prime} ; B\right)+J^{*}\left(A^{\prime}+\left\{-\beta^{*}\right\} ; B^{\prime}\right),
$$

which is the statement of the lemma. 
Now we verify that properties $\mathrm{P} 1$ through $\mathrm{P} 4$ are satisfied in the random matrix situation where we have

$$
Q(S, T)=e^{-N\left(\sum_{\hat{\alpha} \in S} \hat{\alpha}+\sum_{\hat{\beta} \in T} \hat{\beta}\right)} \frac{Z(S, T) Z\left(S^{-}, T^{-}\right)}{Z^{\dagger}\left(S, S^{-}\right) Z^{\dagger}\left(T, T^{-}\right)}
$$

and

$$
H_{S, T}(W)= \begin{cases}\sum_{\hat{\alpha} \in S} \frac{z^{\prime}}{z}(\alpha-\hat{\alpha})-\sum_{\hat{\beta} \in T} \frac{z^{\prime}}{z}(\alpha+\hat{\beta}) & \text { if } W=\{\alpha\} \subset A-S, \\ \sum_{\hat{\beta} \in T} \frac{z^{\prime}}{z}(\beta-\hat{\beta})-\sum_{\hat{\alpha} \in S} \frac{z^{\prime}}{z}(\beta+\hat{\alpha}) & \text { if } W=\{\beta\} \subset B-T, \\ \left(\frac{z^{\prime}}{z}\right)^{\prime}(\alpha+\beta) & \text { if } W=\{\alpha, \beta\} \text { with } \\ 0 & \alpha \in A-S, \beta \in B-T, \\ & \text { otherwise. }\end{cases}
$$

We will start with the simplest case that $\alpha^{*} \in \bar{S}, \beta^{*} \in \bar{T}$. The only polar term from $\alpha^{*}=-\beta^{*}$ arises from a situation when one of the partition parts $W_{r}=\left\{\alpha^{*}, \beta^{*}\right\}$ and there is a pole from $H_{S, T}\left(W_{r}\right)=\left(\frac{z^{\prime}}{z}\right)^{\prime}\left(\alpha^{*}+\beta^{*}\right)$. Since $\left(\frac{z^{\prime}}{z}\right)^{\prime}(x)=1 / x^{2}+\mathrm{O}(1)$ and $\alpha^{*}$ and $\beta^{*}$ do not appear in $Q(S, T)$, this completes the proof of P1.

Next, suppose that $\alpha^{*} \in S$ and $\beta^{*} \in \bar{T}$. The only pole in $D_{S, T}(\bar{S}, \bar{T})$ occurs in the product of the $H$ for $H_{S, T}\left(W_{r}\right)$ when $W_{r}=\left\{\beta^{*}\right\}$. We have

$$
H_{S, T}\left(\left\{\beta^{*}\right\}\right)=\sum_{\hat{\beta} \in T} \frac{z^{\prime}}{z}\left(\hat{\beta}-\beta^{*}\right)-\sum_{\hat{\alpha} \in S} \frac{z^{\prime}}{z}\left(\beta^{*}+\hat{\alpha}\right)
$$

for which, when $\hat{\alpha}=\alpha^{*}$, the term $-\frac{z^{\prime}}{z}\left(\beta^{*}+\alpha^{*}\right)$ has a simple pole at $\alpha^{*}=-\beta^{*}$ with residue $1 . Q(S, T)$ is clearly regular at $\alpha^{*}=-\beta^{*}$.

Next, when $\alpha^{*} \in \bar{S}$ and $\beta^{*} \in T$, the only pole in the product of the $H$ occurs for $H_{S, T}\left(\left\{\alpha^{*}\right\}\right)$. We have

$$
H_{S, T}\left(\left\{\alpha^{*}\right\}\right)=\sum_{\hat{\alpha} \in S} \frac{z^{\prime}}{z}\left(\hat{\alpha}-\alpha^{*}\right)-\sum_{\hat{\beta} \in T} \frac{z^{\prime}}{z}\left(\alpha^{*}+\hat{\beta}\right)
$$


for which, when $\hat{\beta}=\beta^{*}$, the term $-\frac{z^{\prime}}{z}\left(\alpha^{*}+\beta^{*}\right)$ has a simple pole at $\alpha^{*}=-\beta^{*}$ with residue 1 . $Q(S, T)$ does not depend on $\alpha^{*}$.

Finally, we consider the case $\alpha^{*} \in S$ and $\beta^{*} \in T$. We have

$$
Q(S, T)=z\left(\alpha^{*}+\beta^{*}\right) z\left(-\alpha^{*}-\beta^{*}\right) Q_{1}(S, T)
$$

where

$$
Q_{1}(S, T)=Q\left(S^{\prime}, T^{\prime}\right) e^{-N\left(\alpha^{*}+\beta^{*}\right)}
$$

$$
\times \frac{\prod_{\hat{\beta} \in T^{\prime}} z\left(\alpha^{*}+\hat{\beta}\right) z\left(-\alpha^{*}-\hat{\beta}\right) \prod_{\hat{\alpha} \in S^{\prime}} z\left(\hat{\alpha}+\beta^{*}\right) z\left(-\hat{\alpha}-\beta^{*}\right)}{\prod_{\hat{\alpha} \in S^{\prime}} z\left(\alpha^{*}-\hat{\alpha}\right) z\left(\hat{\alpha}-\alpha^{*}\right) \prod_{\hat{\beta} \in T^{\prime}} z\left(\beta^{*}-\hat{\beta}\right) z\left(\hat{\beta}-\beta^{*}\right)} .
$$

Note that

$$
\left.Q_{1}(S, T)\right|_{\alpha^{*}=-\beta^{*}}=Q\left(S^{\prime}, T^{\prime}\right)
$$

We are well on our way to verifying property $\mathrm{P} 4$ because $z\left(\alpha^{*}+\beta^{*}\right) z\left(-\alpha^{*}-\right.$ $\left.\beta^{*}\right)=\frac{-1}{\left(\alpha^{*}+\beta^{*}\right)^{2}}+\frac{1}{12}+\mathrm{O}\left(\left|\alpha^{*}+\beta^{*}\right|\right)$ and we have an expansion for $Q_{1}(S, T)$ in the neighborhood of $\alpha^{*}=-\beta^{*}$ :

$$
\begin{aligned}
Q_{1}(S, T)= & Q\left(S^{\prime}, T^{\prime}\right)\left(1-N\left(\alpha^{*}+\beta^{*}\right)+\mathrm{O}\left(\left|\alpha^{*}+\beta^{*}\right|^{2}\right)\right) \\
& \times\left(1+\left(\alpha^{*}+\beta^{*}\right)\left(\sum_{\hat{\alpha} \in S^{\prime}}\left(\frac{z^{\prime}}{z}\left(\hat{\alpha}+\beta^{*}\right)-\frac{z^{\prime}}{z}\left(-\beta^{*}-\hat{\alpha}\right)\right)\right.\right. \\
& \left.\left.+\sum_{\hat{\beta} \in T^{\prime}}\left(\frac{z^{\prime}}{z}\left(-\beta^{*}+\hat{\beta}\right)-\frac{z^{\prime}}{z}\left(\beta^{*}-\hat{\beta}\right)\right)\right)+\mathrm{O}\left(\left|\alpha^{*}+\beta^{*}\right|^{2}\right)\right) \\
= & Q\left(S^{\prime}, T^{\prime}\right)\left(1-\left(\alpha^{*}+\beta^{*}\right)\left(N+\left.H_{S^{\prime}, T^{\prime}}\left(\left\{\alpha^{*}\right\}\right)\right|_{\alpha^{*}=-\beta^{*}}\right.\right. \\
& \left.\left.+H_{S^{\prime}, T^{\prime}}\left(\left\{\beta^{*}\right\}\right)\right)+\mathrm{O}\left(\left|\alpha^{*}+\beta^{*}\right|^{2}\right)\right) .
\end{aligned}
$$


Now we obtain an expansion for $H_{S, T}(W)$, where we remember that $W \subset \bar{S}+\bar{T}$ and so $W$ does not contain $\alpha^{*}$ or $\beta^{*}$. By (3.48) we have that

$$
\begin{aligned}
H_{S, T}(\{\alpha\}) & =\sum_{\hat{\alpha} \in S} \frac{z^{\prime}}{z}(\alpha-\hat{\alpha})-\sum_{\hat{\beta} \in T} \frac{z^{\prime}}{z}(\alpha+\hat{\beta}) \\
& =H_{S^{\prime}, T^{\prime}}(\{\alpha\})+\frac{z^{\prime}}{z}\left(\alpha-\alpha^{*}\right)-\frac{z^{\prime}}{z}\left(\alpha+\beta^{*}\right) ; \\
H_{S, T}(\{\beta\}) & =\sum_{\hat{\beta} \in T} \frac{z^{\prime}}{z}(\beta-\hat{\beta})-\sum_{\hat{\alpha} \in S} \frac{z^{\prime}}{z}(\beta+\hat{\alpha}) \\
& =H_{S^{\prime}, T^{\prime}}(\{\beta\})+\frac{z^{\prime}}{z}\left(\beta-\beta^{*}\right)-\frac{z^{\prime}}{z}\left(\beta+\alpha^{*}\right) ;
\end{aligned}
$$

and

$$
H_{S, T}(\{\alpha, \beta\})=\left(\frac{z^{\prime}}{z}\right)^{\prime}(\alpha+\beta)=H_{S^{\prime}, T^{\prime}}(\{\alpha, \beta\}) .
$$

Thus,

$$
\left.H_{S, T}(W)\right|_{\alpha^{*}=-\beta^{*}}=H_{S^{\prime}, T^{\prime}}(W)
$$

and

$$
\left.\frac{d}{d \alpha^{*}} H_{S, T}(W)\right|_{\alpha^{*}=-\beta^{*}}= \begin{cases}-\left(\frac{z^{\prime}}{z}\right)^{\prime}\left(\alpha+\beta^{*}\right) & \text { if } W=\{\alpha\}, \\ -\left(\frac{z^{\prime}}{z}\right)^{\prime}\left(\beta-\beta^{*}\right) & \text { if } W=\{\beta\}, \\ 0 & \text { otherwise. }\end{cases}
$$


Note that we can write this as

$$
\left.\frac{d}{d \alpha^{*}} H_{S, T}(W)\right|_{\alpha^{*}=-\beta^{*}}=-\left.H_{S^{\prime}, T^{\prime}}\left(W+\left\{\alpha^{*}\right\}\right)\right|_{\alpha^{*}=-\beta^{*}}-H_{S^{\prime}, T^{\prime}}\left(W+\left\{\beta^{*}\right\}\right),
$$

where one or both of the terms will be zero. From this the expansion of $H_{S, T}(W)$ in $\mathrm{P} 4$ follows.

This concludes the proof of Lemma 3.2.

\section{4. $n$-Correlation via the Ratios theorem}

In this section we will prove the following expression for the $n$-correlation.

Theorem 3.4. Let $\mathcal{C}_{-}$denote the path from $-\delta+\pi i$ down to $-\delta-\pi i$ and let $\mathcal{C}_{+}$denote the path from $\delta-\pi i$ up to $\delta+\pi i$ and let $f$ be a $2 \pi$-periodic, holomorphic function of $n$ variables. Using the notation $J(A ; B)$ from Theorem 3.3,

$$
\begin{aligned}
& \int_{U(N)} \sum_{j_{1}, \ldots, j_{n}=1}^{N} f\left(\theta_{j_{1}}, \ldots, \theta_{j_{n}}\right) d X \\
& =\frac{1}{(2 \pi i)^{n}} \sum_{K+L+M=\{1, \ldots, n\}}(-1)^{|L|+|M|} N^{|M|} \\
& \quad \times \int_{\mathcal{C}_{+}{ }^{K}} \int_{\mathcal{C}_{-}{ }^{L+M}} J\left(z_{K} ;-z_{L}\right) f\left(i z_{1}, \ldots, i z_{n}\right) d z_{1} \ldots d z_{n}
\end{aligned}
$$

where $z_{K}=\left\{z_{k}: k \in K\right\},-z_{L}=\left\{-z_{\ell}: \ell \in L\right\}$ and $\int_{\mathcal{C}_{+} K} \int_{\mathcal{C}_{-}{ }^{L+M}}$ means that we are integrating all of the variables in $z_{K}$ along the $\mathcal{C}_{+}$path and all of the variables in $z_{L}$ or $z_{M}$ along the $\mathcal{C}_{-}$path.

Proof. Since

$$
g(z)=\Lambda_{X}\left(e^{z}\right)=\prod_{j=1}^{N}\left(1-e^{z} e^{-i \theta_{j}}\right)
$$


has zeros at $z=i \theta_{j}+2 \pi i m, m \in \mathbb{Z}$, by Cauchy's theorem we can express a sum

$$
\sum_{j=1}^{N} f\left(\theta_{j}\right)=\frac{1}{2 \pi i} \int_{\mathcal{C}} \frac{g^{\prime}}{g}(z) f(z / i) d z=\frac{1}{2 \pi i} \int_{\mathcal{C}} e^{z} \frac{\Lambda_{X}^{\prime}}{\Lambda_{X}}\left(e^{z}\right) f(z / i) d z
$$

where $\mathcal{C}$ is a positively oriented contour which encloses a subinterval of the imaginary axis of length $2 \pi$. We choose a specific path $\mathcal{C}$ to be the positively oriented rectangle that has vertices $\delta-\pi i, \delta+\pi i,-\delta+\pi i,-\delta-\pi i$, where $\delta$ is a small positive number. More generally, we have

$$
\begin{aligned}
& \sum_{j_{1}, \ldots, j_{n}=1}^{N} f\left(\theta_{j_{1}}, \ldots, \theta_{j_{n}}\right) \\
& =\frac{1}{(2 \pi i)^{n}} \int_{\mathcal{C}} \cdots \int_{\mathcal{C}} \prod_{j=1}^{n} e^{z_{j}} \frac{\Lambda_{X}^{\prime}}{\Lambda_{X}}\left(e^{z_{j}}\right) f\left(z_{1} / i, \ldots, z_{n} / i\right) d z_{1} \cdots d z_{n} .
\end{aligned}
$$

We average this equation over $X \in U(N)$ and, after a change of variables $z_{j} \rightarrow-z_{j}$, we obtain

$$
\begin{aligned}
& \int_{U(N)} \sum_{j_{1}, \ldots, j_{n}=1}^{N} f\left(\theta_{j_{1}}, \ldots, \theta_{j_{n}}\right) d X \\
& \quad=\frac{1}{(2 \pi i)^{n}} \int_{\mathcal{C}^{n}} J\left(z_{1}, \ldots, z_{n} ;\right) f\left(i z_{1}, \ldots, i z_{n}\right) d z_{1} \cdots d z_{n}
\end{aligned}
$$

Let $\mathcal{C}_{-}$denote the path along the left side of $\mathcal{C}$ from $-\delta+\pi i$, down to $-\delta-\pi i$, and let $\mathcal{C}_{+}$denote the path along the right side of $\mathcal{C}$ from $\delta-\pi i$ up to $\delta+\pi i$. Since the periodicity of the function $f$ implies that the horizontal segments of the contours cancel, each variable $z_{j}$ is on one or the other of these two vertical paths. Thus, our expression is a sum of $2^{n}$ terms, each term being an $n$-fold integral with each integral on a vertical line segment either $\mathcal{C}_{-}$or $\mathcal{C}_{+}$. For each variable $z_{j}$ which is on $\mathcal{C}_{-}$we use the functional equation $(2.15)$ to replace $e^{-z_{j}} \frac{\Lambda_{X}^{\prime}}{\Lambda_{X}}\left(e^{-z_{j}}\right)$ by $N-e^{z_{j}} \frac{\Lambda_{X^{*}}^{\prime}}{\Lambda_{X^{*}}}\left(e^{z_{j}}\right)$. In this way 
we find that

$$
\begin{aligned}
& \frac{1}{(2 \pi i)^{n}} \int_{\mathcal{C}^{n}} J\left(z_{1}, \ldots, z_{n} ;\right) f\left(i z_{1}, \ldots, i z_{n}\right) d z_{1} \cdots d z_{n} \\
& =\frac{1}{(2 \pi i)^{n}} \sum_{\epsilon_{j} \in\{-1,+1\}} \int_{\mathcal{C}_{\epsilon_{1}}} \ldots \int_{\mathcal{C}_{\epsilon_{n}}} \int_{U(N)}(-1)^{n} \prod_{j=1}^{n} \\
& \quad \times\left(\frac{1-\epsilon_{j}}{2} N+\epsilon_{j} e^{-\epsilon_{j} z_{j}} \frac{\Lambda_{X^{\epsilon_{j}}}^{\prime}}{\Lambda_{X^{\epsilon_{j}}}}\left(e^{-\epsilon_{j} z_{j}}\right)\right) f\left(i z_{1}, \ldots, i z_{n}\right) d X d z_{1} \cdots d z_{n}
\end{aligned}
$$

Another way to write this equation is

$$
\begin{aligned}
& \frac{1}{(2 \pi i)^{n}} \int_{\mathcal{C}^{n}} J\left(z_{1}, \ldots, z_{n} ;\right) f\left(i z_{1}, \ldots, i z_{n}\right) d z_{1} \cdots d z_{n} \\
& =\frac{1}{(2 \pi i)^{n}} \int_{U(N)}(-1)^{n} \sum_{K \subset\{1, \ldots, n\}} \prod_{j \in K} \int_{\mathcal{C}_{+}} e^{-z_{j}} \frac{\Lambda_{X}^{\prime}}{\Lambda_{X}}\left(e^{-z_{j}}\right) \\
& \quad \times \prod_{j \notin K} \int_{\mathcal{C}_{-}}\left(N-e^{z_{j}} \frac{\Lambda_{X^{*}}^{\prime}}{\Lambda_{X^{*}}}\left(e^{z_{j}}\right)\right) f\left(i z_{1}, \ldots, i z_{n}\right) d z_{1} \cdots d z_{n} d X
\end{aligned}
$$

The expansion of the product over $j \notin K$ can be easily expressed as a sum over further subsets of $\{1, \ldots, n\}$. We have

$$
\begin{aligned}
& \frac{1}{(2 \pi i)^{n}} \int_{\mathcal{C}^{n}} J\left(z_{1}, \ldots, z_{n} ;\right) f\left(i z_{1}, \ldots, i z_{n}\right) d z_{1} \cdots d z_{n} \\
& =\frac{1}{(2 \pi i)^{n}} \int_{U(N)}(-1)^{n} \sum_{K+L+M=\{1, \ldots, n\}} \prod_{j \in K} \int_{\mathcal{C}_{+}} e^{-z_{j}} \frac{\Lambda_{X}^{\prime}}{\Lambda_{X}}\left(e^{-z_{j}}\right)
\end{aligned}
$$

$$
\times \prod_{j \in L} \int_{\mathcal{C}_{-}}(-1) e^{z_{j}} \frac{\Lambda_{X^{*}}^{\prime}}{\Lambda_{X^{*}}}\left(e^{z_{j}}\right) \prod_{j \in M} \int_{\mathcal{C}_{-}} N f\left(i z_{1}, \ldots, i z_{n}\right) d z_{1} \cdots d z_{n} d X
$$

Using this last equation, (3.65) and the definition of $J(A ; B)$ from Theorem 3.3, we have the statement of the theorem.

\section{5. n-Correlation theorem}

We will now prove our main theorem. 
Theorem 3.5. Let $J^{*}$ be as defined in Theorem 3.3. Then

$$
\begin{aligned}
& \int_{U(N))_{1 \leq j_{1}, \ldots, j_{n} \leq N}} \sum_{=}^{*} f\left(\theta_{j_{1}}, \ldots, \theta_{j_{n}}\right) d X_{N} \\
& =\frac{1}{(2 \pi)^{n}} \int_{[0,2 \pi]^{n}} \sum_{K+L+M=\{1, \ldots, n\}} N^{|M|} J^{*}\left(-i \theta_{K} ; i \theta_{L}\right) \\
& \quad \times f\left(\theta_{1}, \ldots, \theta_{n}\right) d \theta_{1} \cdots d \theta_{n},
\end{aligned}
$$

where $i \theta_{L}=\left\{i \theta_{\ell}: \ell \in L\right\},-i \theta_{K}=\left\{-i \theta_{k}: k \in K\right\}$ and the star on the sum indicates summation over distinct indices. Moreover, the integrand has no poles on the path of integration.

This is clearly a far more unwieldy formula for the $n$-correlation than the usual determinantal expression, and as such its calculation might appear to be a pointless exercise. However, the reason for working out this result is that its structure is precisely that of the $n$-correlation of the Riemann zeros, stated in Theorem 4.3. This is yet another illustration of how we can make sense of an impossibly complicated formula from number theory by seeing it as arithmetic decorations hung on the solid framework of a rigorous result from the random matrix theory. This can be seen easily in the examples in Section 5. There we compare formulae where $\zeta(1+x)$ takes the place of the function $z(x)$ and other arithmetic terms appear, but in all its important aspects the structure of the $n$-correlation of the Riemann zeros and that of eigenvalues of random unitary matrices is absolutely identical.

Note the similar forms of Theorems 3.4 and 3.5. In the former the sum is over all indices and the integrals are on paths slightly shifted away from the imaginary axis, and in the latter the sum is over distinct indices and the integration is along the imaginary axis. Moving the integrals onto the imaginary axis results in some principal value terms, and surprisingly these cancel exactly with extra terms in the sum in 3.4.

We actually prove a more general theorem (Theorem 3.6 below). We start with a little notation: For a given $n$ and $0 \leq R \leq n$, let the sum $\sum_{j_{1}, \ldots, j_{n}=1}^{N}$ with the additional condition that $j_{m} \neq j_{\ell}$ if $m>R$ and $\ell>R$ be denoted by $\sum^{n, R}$. If we additionally fix three disjoint sets $K, L$ and $M$ whose union is $\{1,2, \ldots, n\}$, then we introduce the following notation for 
the familiar integral

$$
\begin{aligned}
\int_{-\pi i}^{\pi i} & \cdots \int_{-\pi i}^{\pi i} \int_{C_{+}^{K \cap\{1, \ldots, R\}}} \int_{C_{-}^{(L+M) \cap\{1, \ldots, R\}}} J^{*}\left(z_{K} ;-z_{L}\right) f\left(i z_{1}, \ldots, i z_{n}\right) \\
& \times d z_{1} \cdots d z_{R} d z_{R+1} \cdots d z_{n} \\
= & : I_{f ; K, L, M}^{n, R} .
\end{aligned}
$$

Once again, the integrals on the imaginary axis are principal value integrals.

We have already derived equation (3.61). In the new notation this is written as

$$
\begin{aligned}
& (2 \pi i)^{n} \int_{U(N)} \sum_{K+L+M=\{1, \ldots, n\}}^{n, n} f\left(\theta_{j_{1}}, \ldots, \theta_{j_{n}}\right) d X \\
& \quad=\sum_{K+1)^{|L+M|} N^{|M|} I_{f ; K, L, M}^{n, n} .}(-.)
\end{aligned}
$$

Note that (3.61) features $J$ whereas $I_{f ; K, L, M}^{n, R}$ is defined in terms of $J^{*}$. However, when $R=n$ (that is, all the integrals are off the imaginary axis) Theorem 3.3 says that $J$ and $J^{*}$ are equal.

With the help of Lemma 3.2 we will prove the following:

Theorem 3.6. Using the notation of (3.70) and the preceding paragraph, with $0 \leq R \leq n$,

$$
\begin{aligned}
& (2 \pi i)^{n} \int_{U(N)} \sum^{n, R} f\left(\theta_{j_{1}}, \ldots, \theta_{j_{n}}\right) d X \\
& \quad=\sum_{K+L+M=\{1, \ldots, n\}}(-1)^{|(L+M) \cap\{1, \ldots, R\}|} N^{|M|} I_{f ; K, L, M}^{n, R} .
\end{aligned}
$$

Proof. We will prove this by induction. Assume that Theorem 3.6 holds for $n-1$ and any $0 \leq R \leq n-1$.

We start with the right side of (3.72) and move the $z_{R}$ integral onto the imaginary axis, resulting in a principal value integral and a residue at $z_{R}=z_{t}$, for $t>R$, in any term where $R \in K, t \in L$ or $R \in L, t \in K$. A close inspection of the integral and the form of $J^{*}\left(z_{K} ;-z_{L}\right)$ reveals that there is no pole unless $R$ and $t$ are in one of these two configurations (see Remark 3.2). Also, if $t<R$ then the contour on which $z_{t}$ is integrated has not yet been moved, and so it remains on the far side of the imaginary axis from the $z_{R}$ contour and hence does not yield a pole. Each residue 
contribution comes in the form of the three terms in Lemma 3.2, multiplied by $\pi i$. (It is $\pi i$ rather than $2 \pi i$ because the $z_{R}$ contour is moving precisely onto the imaginary axis, where $z_{t}$ lies, yielding half the contribution of a contour completely encircling the pole.) Thus

$$
\begin{aligned}
& \sum_{K+L+}{ }_{M=\{1, \ldots, n\}}(-1)^{|(L+M) \cap\{1, \ldots, R\}|} N^{|M|} I_{f ; K, L, M}^{n, R} \\
= & \sum_{K+L+M=\{1, \ldots, n\}}(-1)^{|(L+M) \cap\{1, \ldots, R-1\}|} N^{|M|} I_{f ; K, L, M}^{n, R-1} \\
& +2 \times \sum_{t=R+1}^{n} \pi i \sum_{K^{\prime}+L^{\prime}+M=\{1, \ldots, n\}-\{R, t\}}(-1)^{\left|\left(L^{\prime}+M\right) \cap\{1, \ldots, R-1\}\right|} N^{|M|} \\
& \times \int_{-\pi i}^{\pi i} \cdots \int_{-\pi i}^{\pi i} \int_{C_{+}^{K \cap\{1, \ldots, R-1\}}} \int_{C_{-}^{(L+M) \cap\{1, \ldots, R-1\}}}\left(J^{*}\left(z_{K^{\prime}+\{t\}} ;-z_{L^{\prime}}\right)\right. \\
& \left.+J^{*}\left(z_{K^{\prime}} ;-z_{L^{\prime}+\{t\}}\right)+N J^{*}\left(z_{K^{\prime}} ;-z_{L^{\prime}}\right)\right) \\
& \left.\times f\left(i z_{1}, \ldots, i z_{R-1}, i z_{t}, i z_{R+1}, \ldots, i z_{n}\right) d z_{1} \cdots d z_{R-1} d z_{R+1} \cdots d z_{n}\right]
\end{aligned}
$$

The final sum above contains the two identical contributions from the case $R \in K, t \in L$ and the case $R \in L, t \in K$. To confirm the sign of each term, if $R \in K$, the residue is multiplied by $+i \pi$ because the contour of integration moves in from the right of the imaginary axis (skirting the pole in the positive direction) and the argument $z_{R}$ in $J^{*}\left(z_{K} ;-z_{L}\right)$ occurs with a plus sign. Note that $(-1)^{\left|\left(L^{\prime}+M\right) \cap\{1, \ldots, R-1\}\right|}=(-1)^{|(L+M) \cap\{1, \ldots, R\}|}$ if $R \in K$ and $L=L^{\prime}+\{t\}$. On the other hand, if $R \in L$ then the $z_{R}$ contour comes from the left of the imaginary axis, but as $C_{-}$is directed downwards, the pole is still circled in the positive direction. However, $z_{R}$ appears in $J^{*}\left(z_{K} ;-z_{L}\right)$ with a minus sign, so the residue acquires an extra minus sign, which is captured above because $(-1)^{\left|\left(L^{\prime}+M\right) \cap\{1, \ldots, R-1\}\right|}=(-1) \times(-1)^{|(L+M) \cap\{1, \ldots, R\}|}$ if $L=L^{\prime}+\{R\}$.

In the integrals in the final sum above we now relabel the integration variables $z_{1}, z_{2}, \ldots, z_{R-1}, z_{R+1}, \ldots, z_{n}$ by $z_{1}, z_{2}, \ldots, z_{n-1}$ so that $f\left(z_{1}, \ldots, z_{R-1}\right.$, $\left.z_{t}, z_{R+1}, \ldots, z_{t}, \ldots, z_{n}\right)$ is replaced by $f\left(z_{1}, \ldots, z_{R-1}, z_{t-1}, z_{R}, \ldots, z_{t-1}, \ldots\right.$, $\left.z_{n-1}\right)=: g_{t}\left(z_{1}, \ldots, z_{n-1}\right)$. In addition, for some function $h$ of sets $K, L$ 
and $M$,

$$
\begin{aligned}
& \sum_{K+L+M=\{1, \ldots, m-1\}}(h(K+\{m\}, L, M)+h(K, L+\{m\}, M) \\
+ & h(K, L, M+\{m\})) \\
= & \sum_{K+L+M=\{1, \ldots, m\}} h(K, L, M),
\end{aligned}
$$

so we now rewrite the three $J^{*}$ terms in the final sum in (3.73) as a sum over partitions of $\{1, \ldots, n-1\}$. Thus (3.73) equals

$$
\begin{gathered}
\sum_{K+L+M=\{1, \ldots, n\}}(-1)^{|(L+M) \cap\{1, \ldots, R-1\}|} N^{|M|} I_{f ; K, L, M}^{n, R-1} \\
+2 \pi i \sum_{t=R+1}^{n} \sum_{K+L+M=\{1, \ldots, n-1\}}(-1)^{|(L+M) \cap\{1, \ldots, R-1\}|} N^{|M|} I_{g ; K, L, M}^{n-1, R-1} .
\end{gathered}
$$

By the induction hypothesis, this equals

$$
\begin{gathered}
\sum_{K+L+M=\{1, \ldots, n\}}(-1)^{|(L+M) \cap\{1, \ldots, R-1\}|} N^{|M|} I_{f ; K, L, M}^{n, R-1} \\
+2 \pi i \sum_{t=R+1}^{n}(2 \pi i)^{n-1} \int_{U(N)} \sum^{n-1, R-1} g_{t}\left(\theta_{j_{1}}, \ldots, \theta_{j_{n-1}}\right) d X .
\end{gathered}
$$

Note that the left side of $(3.72)$ can be written as

$$
\begin{aligned}
& (2 \pi i)^{n} \int_{U(N)} \sum^{n, R-1} f\left(\theta_{j_{1}}, \ldots, \theta_{j_{n}}\right) d X \\
& +(2 \pi i)^{n} \int_{U(N)} \sum_{t=R+1}^{n} \sum^{n-1, R-1} g_{t}\left(\theta_{j_{1}}, \ldots, \theta_{j_{n-1}}\right) d X
\end{aligned}
$$

where the second sum incorporates all the terms where $\theta_{j_{R}}=\theta_{j_{t}}, t>R$, and then uses the same relabeling of the variables $\theta_{j_{1}}, \theta_{j_{2}}, \ldots, \theta_{j_{R-1}}, \theta_{j_{R+1}}, \ldots, \theta_{j_{n}}$ 
and the definition of $g_{t}$ as described before (3.75). Therefore

$$
\begin{aligned}
& (2 \pi i)^{n} \int_{U(N)} \sum^{n, R-1} f\left(\theta_{j_{1}}, \ldots, \theta_{j_{n}}\right) d X \\
& =\sum_{K+L+M=\{1, \ldots, n\}}(-1)^{|(L+M) \cap\{1, \ldots, R-1\}|} N^{|M|} I_{f ; K, L, M}^{n, R-1}
\end{aligned}
$$

and so, using the induction hypothesis, we have used (3.72) for a given $n$ and $R$ to deduce the same expression for $n$ and $R-1$. Since in (3.71) we have derived the expression for $R=n$ for any $n$, we have shown that if (3.72) is true for $n-1$, it is also true for $n$. To justify the induction hypothesis in $n$, we consider $n=1$. Equation (3.61) states

$$
\begin{aligned}
2 \pi i & \int_{U(N)} \sum^{1,1} f\left(\theta_{j_{1}}\right) d X \\
& =\sum_{K+L+M=\{1\}}(-1)^{|(L+M) \cap\{1\}|} N^{|M|} I_{f ; K, L, M}^{1,1}=-N I_{f ; \emptyset, \emptyset,\{1\}}^{1,1}
\end{aligned}
$$

The final step above follows by remembering that $J^{*}\left(\emptyset ; z_{A}\right)=0$ for any non-empty set $A$. Since $\sum^{1,1}=\sum^{1,0}$ and $I_{f ; \emptyset, \emptyset,\{1\}}^{1,1}=-I_{f ; \emptyset, \emptyset,\{1\}}^{1,0}$, it is immediate that

$$
2 \pi i \int_{U(N)} \sum^{1,0} f\left(\theta_{j_{1}}\right) d X=\sum_{K+L+M=\{1\}}(-1)^{|(L+M) \cap \emptyset|} N^{|M|} I_{f ; K, L, M}^{1,0}
$$

This completes the proof of Theorem 3.6.

It remains to verify that the integrand in Theorem 3.5 has no poles on the path of integration. We have already confirmed in Lemma 3.2 that each $J^{*}\left(-i \theta_{K} ; i \theta_{L}\right)$ has only a simple pole at $\theta_{k}=-\theta_{\ell}$ for $\theta_{k} \in \theta_{K}$ and $\theta_{\ell} \in \theta_{L}$.

We check that

$$
\sum_{K+L+M=\{1,2, \ldots, n\}} N^{M} J^{*}\left(-i \theta_{K} ; i \theta_{L}\right)
$$


has no pole at $\theta_{1}=\theta_{2}$ for generic values of the remaining variables. A given $J^{*}\left(-i \theta_{K} ; i \theta_{L}\right)$ only has a pole when $\theta_{1} \in \theta_{L}$ and $\theta_{2} \in \theta_{K}$, or vice versa, so

$$
\begin{aligned}
& \operatorname{Res}_{\theta_{1}=\theta_{2}} \sum_{K+L+M=\{1,2, \ldots, n\}} N^{M} J^{*}\left(-i \theta_{K} ; i \theta_{L}\right) \\
& =\sum_{K+L+M=\{3, \ldots, n\}} N^{M} \operatorname{Res}_{\theta_{1}=\theta_{2}}\left(J^{*}\left(-i \theta_{K}+\left\{-i \theta_{1}\right\} ;\left\{i \theta_{2}\right\}+i \theta_{L}\right)\right. \\
& \left.\quad+J^{*}\left(-i \theta_{K}+\left\{-i \theta_{2}\right\} ;\left\{i \theta_{1}\right\}+i \theta_{L}\right)\right)=0
\end{aligned}
$$

this is zero because $\operatorname{Res}_{s=x} f(s, x)=-\operatorname{Res}_{s=x} f(x, s)$. Thus if (3.81) had a singular set it would be of complex dimension less than $n-1$ and this implies that there is no singular set (see for example [16, Corollary 7.3.2]).

Our new proof of $n$-correlation in the case of the random matrix theory is now complete.

\section{Correlations of the Riemann zeros}

Now we turn to the Riemann zeta-function. The goal is to obtain a precise conjecture for the $n$-correlation of its zeros and we do this following the method of the previous section for the random matrix case.

\subsection{The Ratios conjecture for the zeta-function}

We derive our formula rigorously from the Ratios conjecture for the zetafunction, which we now state.

Conjecture 4.1 (Ratios Conjecture [6]). Let $Z_{\zeta}(A, B)=\prod_{\substack{\alpha \in A \\ \beta \in B}} \zeta(1+\alpha+$ $\beta)$ and

$$
Z_{\zeta}(A, B ; C, D):=\frac{Z_{\zeta}(A, B) Z_{\zeta}(C, D)}{Z_{\zeta}(A, D) Z_{\zeta}(B, C)}
$$

Further, let

$$
\mathcal{A}_{\zeta}(A, B ; C, D)=\prod_{p} Z_{p}(A, B ; C, D) \int_{0}^{1} \mathcal{A}_{p, \theta}(A, B ; C, D) d \theta
$$


where $z_{p}(x):=\left(1-p^{-x}\right)^{-1}, Z_{p}(A, B)=\prod_{\beta \in A} z_{p \in B}(1+\alpha+\beta)^{-1}$ and

$$
Z_{p}(A, B ; C, D):=\frac{Z_{p}(A, B) Z_{p}(C, D)}{Z_{p}(A, D) Z_{p}(B, C)}
$$

and

$$
\mathcal{A}_{p, \theta}(A, B ; C, D):=\frac{\prod_{\alpha \in A} z_{p,-\theta}(1 / 2+\alpha) \prod_{\beta \in B} z_{p, \theta}(1 / 2+\beta)}{\prod_{\gamma \in C} z_{p,-\theta}(1 / 2+\gamma) \prod_{\delta \in D} z_{p, \theta}(1 / 2+\delta)}
$$

with $z_{p, \theta}(x):=\left(1-e(\theta) p^{-x}\right)^{-1}$. Then, provided that $-\frac{1}{4}<\Re \alpha, \Re \beta<\frac{1}{4}$, $\frac{1}{\log T} \ll \Re \gamma, \Re \delta<\frac{1}{4}$ and $\Im \alpha, \Im \beta, \Im \gamma, \Im \delta \ll T$, we conjecture that, with $s=$ $\frac{1}{2}+i t$, for any interval $I \subset[-T, T]$,

$$
\begin{aligned}
\int_{I} & \frac{\prod_{\alpha \in A} \zeta(s+\alpha) \prod_{\beta \in B} \zeta(1-s+\beta)}{\prod_{\gamma \in C} \zeta(s+\gamma) \prod_{\delta \in D} \zeta(1-s+\delta)} d t \\
& =\int_{I} \mathcal{R}_{\zeta, t}(A, B ; C, D) d t+\mathrm{O}\left(|I|^{1 / 2+\epsilon}\right),
\end{aligned}
$$

where

$$
\mathcal{R}_{\zeta, t}(A, B ; C, D)=\sum_{\substack{S \subset A, T \subset B \\|S|=|T|}} X_{t}(S, T) Z_{\zeta} \mathcal{A}_{\zeta}\left(\bar{S}+T^{-}, \bar{T}+S^{-} ; C, D\right)
$$

Here $T^{-}$means the set of all of the negatives of elements of $T$ (i.e. $T^{-}:=$ $\{-t: t \in T\}), A=S+\bar{S}, B=T+\bar{T}$ and

$$
X_{t}(S, T)=\prod_{\hat{\alpha} \in S} \chi(s+\hat{\alpha}) \prod_{\hat{\beta} \in T} \chi(1-s+\hat{\beta})
$$

where $\chi(1-s)=\chi(s)^{-1}=2(2 \pi)^{-s} \Gamma(s) \cos \frac{\pi s}{2}$ is the factor from the functional equation $\zeta(s)=\chi(s) \zeta(1-s)$.

Remark 4.1. Note that since $|S|=|T|$, for small shifts $\hat{\alpha}$ and $\hat{\beta}$ we have

$$
X_{t}(S, T)=e^{-\ell\left(\sum_{\hat{\alpha} \in S} \hat{\alpha}+\sum_{\hat{\beta} \in T} \hat{\beta}\right)}(1+\mathrm{O}(1 /(1+|t|)),
$$

where $\ell=\log \frac{t}{2 \pi}$, which can sometimes be used to simplify formulae. 
The method for constructing the Ratios conjecture is detailed in [6] and is based on the same principles as the recipe for generating conjectures for moments (see [5]) of zeta and $L$-functions. (Moments cover just the case where $C=\{\emptyset\}$ and $D=\{\emptyset\}$.)

Corollary 4.1. With the same conditions on $\alpha, \beta, \gamma$ and $\delta$ as in Conjecture 4.1 , and with conditions on $\mu$ the same as those on $\alpha$ and $\beta$, we have

$$
\begin{aligned}
& \int_{I} \frac{\prod_{\alpha \in A} \zeta(s+\alpha) \prod_{\beta \in B} \zeta(1-s+\beta)}{\prod_{\gamma \in C} \zeta(s+\gamma) \prod_{\delta \in D} \zeta(1-s+\delta)} \prod_{\mu \in U} \frac{\chi^{\prime}}{\chi}(s+\mu) d t \\
& \quad=\int_{I} \mathcal{R}_{\zeta, t}(A, B ; C, D) \prod_{\mu \in U} \frac{\chi^{\prime}}{\chi}(s+\mu) d t+O\left(|I|^{1 / 2+\epsilon}\right)
\end{aligned}
$$

as a consequence of Conjecture 4.1.

Proof. This follows immediately by integration by parts using the fact that

$$
\frac{\chi^{\prime}}{\chi}(s) \ll \log (2+|s|) \quad \text { and } \quad \frac{d}{d s} \frac{\chi^{\prime}}{\chi}(s) \ll \frac{1}{1+|s|} \text {. }
$$

\subsection{Averages of logarithmic derivatives of the Riemann zeta-function}

To determine the correlations of the Riemann zeros, we will need a result about averaging logarithmic derivatives of the zeta-function:

Theorem 4.1. Assuming the Ratios conjecture, if $\Re \alpha_{i}, \Re \beta_{j}>0$ for $\alpha_{i} \in A$ and $\beta_{j} \in B$ then $J_{\zeta, I}(A ; B ; U)=J_{\zeta, I}^{*}(A ; B ; U)+\mathrm{O}\left(|I|^{1 / 2+\epsilon}\right)$, where for an interval $I$

$$
\begin{aligned}
& J_{\zeta, I}(A ; B ; U) \\
& \quad:=\int_{I} \prod_{\alpha \in A} \frac{\zeta^{\prime}}{\zeta}\left(\frac{1}{2}+i t+\alpha\right) \prod_{\beta \in B} \frac{\zeta^{\prime}}{\zeta}\left(\frac{1}{2}-i t+\beta\right) \prod_{\mu \in U}\left(-\frac{\chi^{\prime}}{\chi}\left(\frac{1}{2}+i t+\mu\right)\right) d t
\end{aligned}
$$


and

$$
J_{\zeta, I}^{*}(A ; B ; U):=\int_{I} J_{\zeta, t}^{*}(A ; B ; U) d t
$$

where

$$
\begin{aligned}
J_{\zeta, t}^{*}(A ; B ; U):= & \sum_{\substack{S \subset A, T \subset B \\
|S|=|T|}} X_{t}(S, T) \frac{Z_{\zeta}(S, T) Z_{\zeta}\left(S^{-}, T^{-}\right)}{Z_{\zeta}^{\dagger}\left(S, S^{-}\right) Z_{\zeta}^{\dagger}\left(T, T^{-}\right)} \mathcal{A}_{\zeta}\left(T^{-}, S^{-} ; S, T\right) \\
& \times \sum_{\substack{\bar{S}+\bar{T} \\
\\
\\
\text { 4.13) }}} \prod_{r=1}^{R} \mathcal{H}_{S, T}\left(W_{r}\right) \times \prod_{\mu \in U}\left(-\frac{\chi^{\prime}}{\chi}(1 / 2+i t+\mu)\right) .
\end{aligned}
$$

Here we use the notation $Z_{\zeta}$ as in Conjecture 4.1 and $Z_{\zeta}^{\dagger}(A, B)=\prod_{\substack{\alpha \in A \\ \beta \in B}}$ $\zeta(1+\alpha+\beta)$. In addition, $T^{-}:=\{-t: t \in T\}, A=S+\bar{S}, B=T+\frac{\alpha+\beta \neq 0}{T}$ and (4.14) $\mathcal{H}_{S, T}\left(W_{r}\right)=H_{\zeta ; S, T}\left(W_{r}\right)-\sum_{p} H_{p, 1 ; S, T}\left(W_{r}\right)+\sum_{p} H_{p, 2 ; S, T}\left(W_{r}\right)$.

Further, we have

$$
H_{\zeta, S, T}(W)=\left\{\begin{array}{cc}
\sum_{\hat{\alpha} \in S} \frac{\zeta^{\prime}}{\zeta}(1+\alpha-\hat{\alpha}) & \\
-\sum_{\hat{\beta} \in T} \frac{\zeta^{\prime}}{\zeta}(1+\alpha+\hat{\beta}) & \text { if } W=\{\alpha\} \subset \bar{S}, \\
\sum_{\hat{\beta} \in T} \frac{\zeta^{\prime}}{\zeta}(1+\beta-\hat{\beta}) & \text { if } W=\{\beta\} \subset \bar{T}, \\
-\sum_{\hat{\alpha} \in S} \frac{\zeta^{\prime}}{\zeta}(1+\beta+\hat{\alpha}) & \text { if } W=\{\alpha, \beta\} \text { with } \\
\left(\frac{\zeta^{\prime}}{\zeta}\right)^{\prime}(1+\alpha+\beta) & \alpha \in \bar{S}, \beta \in \bar{T}, \\
0 & \text { otherwise; }
\end{array}\right.
$$




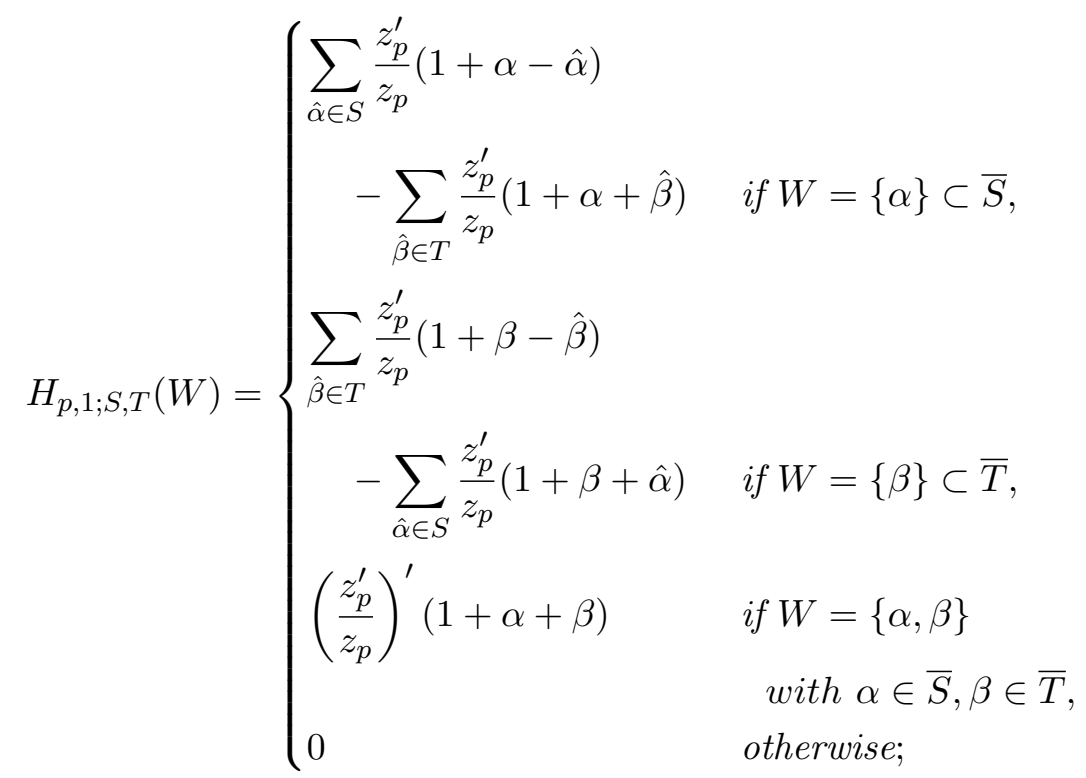

and

$$
H_{p, 2, S, T}(W)=\sum_{W=\sum_{j=1}^{J} X_{j}}(-1)^{J-1}(J-1) ! \prod_{j=1}^{J} c_{S, T}\left(X_{j}\right)
$$

with

$$
c_{S, T}(X):=\frac{\int_{0}^{1} \mathcal{A}_{p, \theta}(S, T) \prod_{\alpha \in \bar{S} \cap X}\left(z_{p,-\theta}^{\prime} / z_{p,-\theta}\right)\left(\frac{1}{2}+\alpha\right) \prod_{\beta \in \bar{T} \cap X}}{\left(z_{p, \theta}^{\prime} / z_{p, \theta}\right)\left(\frac{1}{2}+\beta\right) d \theta}
$$

and the notation

$$
\mathcal{A}_{p, \theta}(S, T):=\mathcal{A}_{p, \theta}\left(T^{-}, S^{-} ; S, T\right),
$$

with $A_{p, \theta}(A, B ; C, D)$ as in Conjecture 4.1.

To prove this we want to differentiate $\mathcal{R}_{\zeta, t}$ with respect to all of the $\alpha \in A$ and $\beta \in B$ and then replace each $\gamma$ by an $\alpha$ and each $\delta$ by a $\beta$. In what follows, $A$ and $C$ have the same cardinality, as do $B$ and $D$. Thus, after differentiation, when we want to set the $\gamma$ equal to the $\alpha$ in some order, and the $\delta$ equal to the $\beta$ in some order, we can abbreviate this by $C=A$ 
and $D=B$. With the definition of $J_{\zeta, I}(A ; B ; U)$ as in $(4.11)$, and using Corollary 4.1, we have

$$
\begin{aligned}
& J_{\zeta, I}(A ; B ; U) \\
& =\left.\int_{\substack { I \\
\begin{subarray}{c}{\alpha \in A \\
\beta \in B{ I \\
\begin{subarray} { c } { \alpha \in A \\
\beta \in B } }\end{subarray}} \frac{d}{d \alpha} \frac{d}{d \beta} \mathcal{R}_{\zeta, t}(A, B ; C, D)\right|_{\substack{C=A \\
D=B}} \prod_{\mu \in U}\left(-\frac{\chi^{\prime}}{\chi}(1 / 2+i t+\mu)\right) d t \\
& \quad+\mathrm{O}\left(|I|^{1 / 2+\epsilon}\right) .
\end{aligned}
$$

The situation is much as in the random matrix theory case, except that now we have to understand how to include the arithmetical factor $\mathcal{A}_{\zeta}$. For a start, we can differentiate with respect to the $\hat{\alpha} \in S$ and $\hat{\beta} \in T$ as before. $C_{\bar{S}}$ and $D_{\bar{T}}$ are defined as in the proof of Theorem 3.3. We have

$$
\begin{aligned}
J_{\zeta, I}(A ; B ; U)= & \int_{I} \prod_{\mu \in U}\left(-\frac{\chi^{\prime}}{\chi}(1 / 2+i t+\mu)\right) \\
& \times \sum_{\substack{S \subset A, T \subset B \\
|S|=|T|}} X_{t}(S, T) \frac{Z_{\zeta}(S, T) Z_{\zeta}\left(S^{-}, T^{-}\right)}{Z_{\zeta}^{\prime}\left(S, S^{-}\right) Z_{\zeta}^{\prime}\left(T, T^{-}\right)} \prod_{\substack{\alpha \in \bar{S} \\
\beta \in \bar{T}}} \frac{d}{d \alpha} \frac{d}{d \beta} \\
& \times\left(\frac{Z_{\zeta}(\bar{S}, \bar{T}) Z_{\zeta}\left(\bar{S}, S^{-}\right) Z_{\zeta}\left(\bar{T}, T^{-}\right) Z(C, D)}{Z_{\zeta}(S, T) Z_{\zeta}\left(C_{\bar{S}}, S^{-}\right) Z_{\zeta}\left(D_{\bar{T}}, T^{-}\right) Z_{\zeta}(\bar{S}, D) Z_{\zeta}(\bar{T}, C)}\right. \\
& \left.\times \mathcal{A}_{\zeta}\left(\bar{S}+T^{-}, \bar{T}+S^{-} ; C, D\right)\right)\left.\right|_{\substack{C=A \\
D=B}} d t+\mathrm{O}\left(|I|^{1 / 2+\epsilon}\right) .
\end{aligned}
$$

In anticipation of applying Lemma 3.1, we note that a brief calculation shows that

$$
\begin{aligned}
& \frac{Z_{\zeta}(\bar{S}, \bar{T}) Z_{\zeta}\left(\bar{S}, S^{-}\right) Z_{\zeta}\left(\bar{T}, T^{-}\right) Z(C, D)}{Z_{\zeta}(S, T) Z_{\zeta}\left(C_{\bar{S}}, S^{-}\right) Z_{\zeta}\left(D_{\bar{T}}, T^{-}\right) Z_{\zeta}(\bar{S}, D) Z_{\zeta}(\bar{T}, C)} \\
& \times\left.\mathcal{A}_{\zeta}\left(\bar{S}+T^{-}, \bar{T}+S^{-} ; C, D\right)\right|_{\substack{C=A \\
D=B}} \\
& =\mathcal{A}_{\zeta}\left(T^{-}, S^{-} ; S, T\right) .
\end{aligned}
$$


The remainder of the proof of Theorem 4.1 consists of applying Lemma 3.1 with (keeping just the factors from the big brackets in (4.21) that depend on $\bar{S}$ and $\bar{T}$ )

$$
\begin{aligned}
H= & \log Z_{\zeta}(\bar{S}, \bar{T})+\log Z_{\zeta}\left(\bar{S}, S^{-}\right)+\log Z_{\zeta}\left(\bar{T}, T^{-}\right)-\log Z_{\zeta}(\bar{S}, D) \\
& -\log Z_{\zeta}(\bar{T}, C)+\sum_{p}\left(\log Z_{p}(\bar{S}, \bar{T})+\log Z_{p}\left(\bar{S}, S^{-}\right)+\log Z_{p}\left(\bar{T}, T^{-}\right)\right. \\
& -\log Z_{p}(\bar{S}, D)-\log Z_{p}(\bar{T}, C)+\log \int_{0}^{1} \mathcal{A}_{p, \theta}\left(\bar{S}+T^{-}, \bar{T}+S^{-} ; C, D\right) d \theta
\end{aligned}
$$

Note the exponent -1 in the definition of $Z_{p}(A, B)$ in Conjecture 4.1 which accounts for the minus sign in front of $H_{1, p, S, T}\left(W_{r}\right)$ in the definition in (4.14) of $H_{S, T}\left(W_{r}\right)$.

Now it just remains to prove:

Lemma 4.1. Let $W \subset \bar{S}+\bar{T}$. Then

$$
\begin{aligned}
H_{p, 2, S, T}(W) & :=\left.\prod_{w \in W} \frac{d}{d w} \log \left(\int_{0}^{1} \mathcal{A}_{p, \theta}\left(\bar{S}+T^{-}, \bar{T}+S^{-} ; C, D\right) d \theta\right)\right|_{\substack{C=\bar{S}+S \\
D=\bar{T}+T}} \\
& =\sum_{W=\sum_{j=1}^{J} X_{j}}(-1)^{J-1}(J-1) ! \prod_{j=1}^{J} c_{S, T}\left(X_{j}\right) ;
\end{aligned}
$$

here

$$
c_{S, T}(X):=\frac{\begin{array}{c}
\int_{0}^{1} \mathcal{A}_{p, \theta}(S, T) \prod_{\alpha \in \bar{S} \cap X}\left(z_{p,-\theta}^{\prime} / z_{p,-\theta}\right)\left(\frac{1}{2}+\alpha\right) \\
\times \prod_{\beta \in \bar{T} \cap X}\left(z_{p, \theta}^{\prime} / z_{p, \theta}\right)(1 / 2+\beta) d \theta
\end{array}}{\int_{0}^{1} \mathcal{A}_{p, \theta}(S, T) d \theta}
$$

where we have adopted the notation

$$
\mathcal{A}_{p, \theta}(S, T):=\mathcal{A}_{p, \theta}\left(T^{-}, S^{-} ; S, T\right) .
$$


Further, we have, for $\alpha^{*} \in S, \beta^{*} \in T, S^{\prime}=S-\left\{\alpha^{*}\right\}, T^{\prime}=T-\left\{\beta^{*}\right\}$ and $W \subset \bar{S}+\bar{T}$,

$$
\begin{aligned}
\left.\frac{d}{d \alpha^{*}} c_{S, T}(W)\right|_{\alpha^{*}=-\beta^{*}}= & -\left.c_{S^{\prime}, T^{\prime}}\left(W+\left\{\alpha^{*}\right\}\right)\right|_{\alpha^{*}=-\beta^{*}}-c_{S^{\prime}, T^{\prime}}\left(W+\left\{\beta^{*}\right\}\right) \\
& +\left.c_{S^{\prime}, T^{\prime}}(W) c_{S^{\prime}, T^{\prime}}\left(\left\{\alpha^{*}\right\}\right)\right|_{\alpha^{*}=-\beta^{*}}+c_{S^{\prime}, T^{\prime}}(W) \\
& \times c_{S^{\prime}, T^{\prime}}\left(\left\{\beta^{*}\right\}\right)
\end{aligned}
$$

and

$$
\begin{aligned}
\left.\frac{d}{d \alpha^{*}} H_{p, 2 ; S, T}(W)\right|_{\alpha^{*}=-\beta^{*}}= & -\left.H_{p, 2, S^{\prime}, T^{\prime}}\left(W+\left\{\alpha^{*}\right\}\right)\right|_{\alpha^{*}=-\beta^{*}} \\
& -H_{p, 2, S^{\prime}, T^{\prime}}\left(W+\left\{\beta^{*}\right\}\right) .
\end{aligned}
$$

Proof. The proof is simple, involving only differentiation. The first line, (4.24), follows from logarithmic differentiation of the integral of $\mathcal{A}_{p, \theta}$, where each variable $w \in W$ appears in just one place. Equation (4.27) also arises immediately by following the rules of differentiation. Note that $\alpha^{*}$ appears in $\mathcal{A}_{p, \theta}(S, T)$ in the numerator of (4.4) in a factor $z_{p, \theta}\left(\frac{1}{2}-\alpha^{*}\right)$ and in the denominator in a factor of type $z_{p,-\theta}\left(\frac{1}{2}+\alpha^{*}\right)$ and $\alpha^{*} \notin W$. The first term in (4.27) comes from the $z_{p,-\theta}\left(\frac{1}{2}+\alpha^{*}\right)$ factor in the numerator of $(4.25)$, the second term in (4.27) from the $z_{p, \theta}\left(\frac{1}{2}-\alpha^{*}\right)$ and the final two terms in (4.27) from the integral in the denominator of (4.25). To obtain (4.28) note that the $\alpha^{*}$ appears in each factor of $c_{S, T}\left(X_{j}\right), j=1, \ldots, J$, in (4.24). Using the product rule on each term in (4.24) we differentiate each $c_{s, T}\left(X_{j}\right)$ in turn and sum the results. Note that $\left.c_{S, T}\left(X_{j}\right)\right|_{\alpha^{*}=-\beta^{*}}=c_{S^{\prime}, T^{\prime}}\left(X_{j}\right)$. Using (4.27) and careful combinatorial accounting (4.28) can be obtained.

Remark 4.2. We have allowed here some loose use of notation in writing $H_{p, 2, S^{\prime}, T^{\prime}}\left(W+\left\{\alpha^{*}\right\}\right)$. The lemma starts out by defining $H_{p, 2, S, T}(W)$, where $W \subset \bar{S}+\bar{T}$, but of course $W+\left\{\alpha^{*}\right\} \notin \bar{S}+\bar{T}$ when $\alpha^{*} \in S$. However to understand the notation $H_{p, 2, S^{\prime}, T^{\prime}}\left(W+\left\{\alpha^{*}\right\}\right)$ simply replace $S$ with $S^{\prime}$ and $T$ with $T^{\prime}$ in the definition of $H_{p, 2, S, T}$ and replace $\bar{S}$ with $A-S^{\prime}$ and $\bar{T}$ with $B-T^{\prime}$. A similar comment applies to $c_{S^{\prime}, T^{\prime}}\left(W+\left\{\alpha^{*}\right\}\right)$ in (4.27).

\subsection{Residue identity revisited}

Using Theorem 4.1, which is an application of Lemma 3.1 along much the same lines as Theorem 3.3, we now prove the analog for $\zeta$ of Lemma 3.2. 
Lemma 4.2. Suppose that $\alpha^{*} \in A$ and $\beta^{*} \in B$. Let $A^{\prime}=A-\left\{\alpha^{*}\right\}$ and $B^{\prime}=B-\left\{\beta^{*}\right\}$ and $\ell=\log \frac{t}{2 \pi}$. Then $J_{\zeta, t}^{*}(A ; B ; U)$ (defined in Theorem 4.1) has a simple pole at $\alpha^{*}=-\beta^{*}$ with

$$
\begin{aligned}
\underset{\alpha^{*}=-\beta^{*}}{\operatorname{Res}} J_{\zeta, t}^{*}(A ; B ; U)= & -\frac{\chi^{\prime}}{\chi}\left(s-\beta^{*}\right) J_{\zeta, t}^{*}\left(A^{\prime} ; B^{\prime} ; U\right)+J_{\zeta, t}^{*}\left(A^{\prime} ; B ; U\right) \\
& +J_{\zeta, t}^{*}\left(A^{\prime}+\left\{-\beta^{*}\right\} ; B^{\prime} ; U\right) .
\end{aligned}
$$

Proof. We use Lemma 3.2. First we remember the convention that $A=S+\bar{S}$ and $B=T+\bar{T}$ and we write Theorem 4.1 as

$$
J_{\zeta, t}^{*}(A, B ; U)=\prod_{\mu \in U} \frac{\chi^{\prime}}{\chi}(1 / 2+i t+\mu) \sum_{\substack{S \subset A \\ T \subset B \\|S|=|T|}} D_{\zeta ; S, T}(\bar{S}, \bar{T}),
$$

where with the abbreviation $\mathcal{A}(S, T):=\mathcal{A}_{\zeta}\left(T^{-}, S^{-} ; S, T\right)$, we have

$$
D_{\zeta ; S, T}(\bar{S}, \bar{T})=Q_{\zeta}(S, T) \mathcal{A}(S, T) \sum_{\bar{S}+\bar{T}=\sum W_{r}} \prod_{r=1}^{R} \mathcal{H}_{S, T}\left(W_{r}\right)
$$

and

$$
Q_{\zeta}(S, T):=X_{t}(S, T) \frac{Z_{\zeta}(S, T) Z_{\zeta}\left(S^{-}, T^{-}\right)}{Z_{\zeta}^{\dagger}\left(S, S^{-}\right) Z_{\zeta}^{\dagger}\left(T, T^{-}\right)} .
$$

Now we let $Q_{\zeta}(S, T) \mathcal{A}(S, T)$ play the role of $Q(S, T)$ in the proof of Lemma 3.2 and $\mathcal{H}_{S, T}(W)$ plays the role of $H_{S, T}(W)$. Thus we need to prove the four conditions below, describing the behavior of the various components of the formula as $\alpha^{*}$ approaches $-\beta^{*}$, and then the rest of the proof is identical to that of Lemma 3.2.

Q1: If $\alpha^{*} \in \bar{S}$ and $\beta^{*} \in \bar{T}$, then $Q_{\zeta}(S, T) \mathcal{A}(S, T)$ is independent of $\alpha^{*}$ and $\beta^{*}$ and

$$
\mathcal{H}_{S, T}(W)= \begin{cases}\frac{1}{\left(\alpha^{*}+\beta^{*}\right)^{2}}+\mathrm{O}(1) & \text { if } W=\left\{\alpha^{*}, \beta^{*}\right\} \\ \mathrm{O}(1) & \text { otherwise. }\end{cases}
$$


Q2: If $\alpha^{*} \in S$ and $\beta^{*} \in \bar{T}$, then $Q_{\zeta}(S, T) \mathcal{A}(S, T)$ is regular when $\alpha^{*}=-\beta^{*}$ and

$$
\mathcal{H}_{S, T}(W)= \begin{cases}\frac{1}{\alpha^{*}+\beta^{*}}+\mathrm{O}(1) & \text { if } W=\left\{\beta^{*}\right\} \\ \mathrm{O}(1) & \text { otherwise. }\end{cases}
$$

Q3: If $\alpha^{*} \in \bar{S}$ and $\beta^{*} \in T$, then $Q_{\zeta}(S, T) \mathcal{A}(S, T)$ is regular when $\alpha^{*}=-\beta^{*}$ and

$$
\mathcal{H}_{S, T}(W)= \begin{cases}\frac{1}{\alpha^{*}+\beta^{*}}+\mathrm{O}(1) & \text { if } W=\left\{\alpha^{*}\right\} \\ \mathrm{O}(1) & \text { otherwise. }\end{cases}
$$

Q4: If $\alpha^{*} \in S$ and $\beta^{*} \in T$ and $S^{\prime}=S-\left\{\alpha^{*}\right\}$ and $T^{\prime}=T-\left\{\beta^{*}\right\}$, then $Q_{\zeta}(S, T)=\left(\frac{-1}{\left(\alpha^{*}+\beta^{*}\right)^{2}}+\mathrm{O}(1)\right) Q_{\zeta ; 1}(S, T)$ and

$$
\begin{aligned}
Q_{\zeta ; 1}(S, T) \mathcal{A}(S, T)= & Q_{\zeta}\left(S^{\prime}, T^{\prime}\right) \mathcal{A}\left(S^{\prime}, T^{\prime}\right)\left(1-\left(\alpha^{*}+\beta^{*}\right)\left(-\frac{\chi^{\prime}}{\chi}\left(s-\beta^{*}\right)\right.\right. \\
& \left.\left.+\left.\mathcal{H}_{S^{\prime}, T^{\prime}}\left(\left\{\alpha^{*}\right\}\right)\right|_{\alpha^{*}=-\beta^{*}}+\mathcal{H}_{S^{\prime}, T^{\prime}}\left(\left\{\beta^{*}\right\}\right)\right)\right)+\mathrm{O}(1)
\end{aligned}
$$

and

$$
\begin{aligned}
\mathcal{H}_{S, T}(W)= & \mathcal{H}_{S^{\prime}, T^{\prime}}(W)-\left(\alpha^{*}+\beta^{*}\right)\left(\left.\mathcal{H}_{S^{\prime}, T^{\prime}}\left(W+\left\{\alpha^{*}\right\}\right)\right|_{\alpha^{*}=-\beta^{*}}\right. \\
& \left.+\mathcal{H}_{S^{\prime}, T^{\prime}}\left(W+\left\{\beta^{*}\right\}\right)\right)+O\left(\left|\alpha^{*}+\beta^{*}\right|^{2}\right) .
\end{aligned}
$$

We have just to prove these four conditions to complete the proof. We start with the first case where $\alpha^{*} \notin S, \beta^{*} \notin T$. Then $\alpha^{*} \in \bar{S}$ and $\beta^{*} \in \bar{T}$. The terms $H_{p, 1}$ and $H_{p, 2}$ have no poles because of the conditions on the real parts of $\alpha$ and $\beta$. The only polar term from $\alpha^{*}=-\beta^{*}$ arises from a situation when one of the partition parts is $W_{r}=\left\{\alpha^{*}, \beta^{*}\right\}$ and there is a pole from $H_{\zeta ; S, T}\left(W_{r}\right)=\left(\frac{\zeta^{\prime}}{\zeta}\right)^{\prime}\left(1+\alpha^{*}+\beta^{*}\right)$. Since $\left(\frac{\zeta^{\prime}}{\zeta}\right)^{\prime}(1+x)=1 / x^{2}+\mathrm{O}(1)$ and $Q_{\zeta}(S, T) \mathcal{A}(S, T)$ is clearly independent of $\alpha^{*}$ and $\beta^{*}$, the first condition is satisfied.

Next, suppose that $\alpha^{*} \in S$ and $\beta^{*} \notin T$. The only pole in $D_{\zeta ; S, T}(\bar{S}, \bar{T})$ occurs in the product of the $H$ for $H_{\zeta ; S, T}\left(W_{r}\right)$ when $W_{r}=\left\{\beta^{*}\right\}$. We have

$$
H_{\zeta ; S, T}\left(\left\{\beta^{*}\right\}\right)=\sum_{\hat{\beta} \in T} \frac{\zeta^{\prime}}{\zeta}\left(1+\hat{\beta}-\beta^{*}\right)-\sum_{\hat{\alpha} \in S} \frac{\zeta^{\prime}}{\zeta}\left(1+\beta^{*}+\hat{\alpha}\right)
$$


for which, when $\hat{\alpha}=\alpha^{*}$, the term $-\frac{\zeta^{\prime}}{\zeta}\left(1+\beta^{*}+\alpha^{*}\right)$ has a simple pole at $\alpha^{*}=-\beta^{*}$ with residue $1 . Q_{\zeta}(S, T) \mathcal{A}(S, T)$ depends on $\alpha^{*}$ and not $\beta^{*}$, so it is regular when $\alpha^{*}=-\beta^{*}$.

Similarly, when $\alpha^{*} \notin S$ and $\beta^{*} \in T$, the only pole in the product of the $H$ occurs for $H_{\zeta ; S, T}\left(\left\{\alpha^{*}\right\}\right)$. We have

$$
H_{\zeta ; S, T}\left(\left\{\alpha^{*}\right\}\right)=\sum_{\hat{\alpha} \in S} \frac{\zeta^{\prime}}{\zeta}\left(1+\hat{\alpha}-\alpha^{*}\right)-\sum_{\hat{\beta} \in T} \frac{\zeta^{\prime}}{\zeta}\left(1+\alpha^{*}+\hat{\beta}\right)
$$

for which, when $\hat{\beta}=\beta^{*}$, the term $-\frac{\zeta^{\prime}}{\zeta}\left(1+\alpha^{*}+\beta^{*}\right)$ has a simple pole at $\alpha^{*}=-\beta^{*}$ with residue 1 .

Finally, we consider the case $\alpha^{*} \in S$ and $\beta^{*} \in T$. Let $S^{\prime}=S-\left\{\alpha^{*}\right\}$ and $T^{\prime}=T-\left\{\beta^{*}\right\}$. We have

$$
Q_{\zeta}(S, T)=\zeta\left(1+\alpha^{*}+\beta^{*}\right) \zeta\left(1-\alpha^{*}-\beta^{*}\right) Q_{\zeta ; 1}(S, T)
$$

where

$$
Q_{\zeta ; 1}(S, T)=Q_{\zeta}\left(S^{\prime}, T^{\prime}\right) X_{t}\left(\left\{\alpha^{*}\right\},\left\{\beta^{*}\right\}\right)
$$

$$
\times \frac{\prod_{\hat{\beta} \in T^{\prime}} \zeta\left(1+\alpha^{*}+\hat{\beta}\right) \zeta\left(1-\alpha^{*}-\hat{\beta}\right) \prod_{\hat{\alpha} \in S^{\prime}} \zeta\left(1+\hat{\alpha}+\beta^{*}\right) \zeta\left(1-\hat{\alpha}-\beta^{*}\right)}{\prod_{\hat{\alpha} \in S^{\prime}} \zeta\left(1+\alpha^{*}-\hat{\alpha}\right) \zeta\left(1+\hat{\alpha}-\alpha^{*}\right) \prod_{\hat{\beta} \in T^{\prime}} \zeta\left(1+\beta^{*}-\hat{\beta}\right) \zeta\left(1+\hat{\beta}-\beta^{*}\right)} .
$$

Note that $\zeta\left(1+\alpha^{*}+\beta^{*}\right) \zeta\left(1-\alpha^{*}-\beta^{*}\right)=\frac{-1}{\left(\alpha^{*}+\beta^{*}\right)^{2}}+\mathrm{O}(1)$. Also, remembering that $\chi\left(s-\beta^{*}\right) \chi\left(1-s+\beta^{*}\right)=1$,

$$
\left.Q_{\zeta ; 1}(S, T)\right|_{\alpha^{*}=-\beta^{*}}=Q_{\zeta}\left(S^{\prime}, T^{\prime}\right)
$$

which gives us an expansion for $Q_{\zeta ; 1}(S, T)$ in the neighborhood of $\alpha^{*}=-\beta^{*}$ :

$$
\begin{aligned}
Q_{\zeta ; 1}(S, T)= & Q_{\zeta}\left(S^{\prime}, T^{\prime}\right)\left(1+\frac{\chi^{\prime}}{\chi}\left(s-\beta^{*}\right)\left(\alpha^{*}+\beta^{*}\right)+\mathrm{O}\left(\left|\alpha^{*}+\beta^{*}\right|^{2}\right)\right. \\
& \times\left(1+\left(\alpha^{*}+\beta^{*}\right)\left(\sum_{\hat{\alpha} \in S^{\prime}}\left(\frac{\zeta^{\prime}}{\zeta}\left(1+\hat{\alpha}+\beta^{*}\right)-\frac{\zeta^{\prime}}{\zeta}\left(1-\beta^{*}-\hat{\alpha}\right)\right)\right.\right. \\
& \left.\left.+\sum_{\hat{\beta} \in T^{\prime}}\left(\frac{\zeta^{\prime}}{\zeta}\left(1-\beta^{*}+\hat{\beta}\right)-\frac{\zeta^{\prime}}{\zeta}\left(1+\beta^{*}-\hat{\beta}\right)\right)\right)+\mathrm{O}\left(\left|\alpha^{*}+\beta^{*}\right|^{2}\right)\right)
\end{aligned}
$$




$$
\begin{aligned}
& =Q_{\zeta}\left(S^{\prime}, T^{\prime}\right)\left(1-\left(\alpha^{*}+\beta^{*}\right)\left(-\frac{\chi^{\prime}}{\chi}\left(s-\beta^{*}\right)\right.\right. \\
& \left.\left.+\left.H_{\zeta ; S^{\prime}, T^{\prime}}\left(\left\{\alpha^{*}\right\}\right)\right|_{\alpha^{*}=-\beta^{*}}+H_{\zeta ; S^{\prime}, T^{\prime}}\left(\left\{\beta^{*}\right\}\right)\right)+O\left(\left|\alpha^{*}+\beta^{*}\right|^{2}\right)\right) .
\end{aligned}
$$

Since $\left.\mathcal{A}(S, T)\right|_{\alpha^{*}=-\beta^{*}}=\mathcal{A}\left(S^{\prime}, T^{\prime}\right)$, we have the expansion around $\alpha^{*}=-\beta^{*}$ :

$$
\begin{aligned}
\mathcal{A}(S, T)= & \mathcal{A}\left(S^{\prime}, T^{\prime}\right)\left(1+\left(\alpha^{*}+\beta^{*}\right) \sum_{p}\left(\sum _ { \hat { \alpha } \in S ^ { \prime } } \left(-\frac{z_{p}^{\prime}}{z_{p}}\left(1+\hat{\alpha}+\beta^{*}\right)\right.\right.\right. \\
& \left.+\frac{z_{p}^{\prime}}{z_{p}}\left(1-\beta^{*}-\hat{\alpha}\right)\right)+\sum_{\hat{\beta} \in T^{\prime}}\left(-\frac{z_{p}^{\prime}}{z_{p}}\left(1-\beta^{*}+\hat{\beta}\right)+\frac{z_{p}^{\prime}}{z_{p}}\left(1+\beta^{*}-\hat{\beta}\right)\right) \\
& \left.\left.-\frac{\int_{0}^{1} \mathcal{A}_{p, \theta}\left(S^{\prime}, T^{\prime}\right)\left(\left(z_{p,-\theta}^{\prime} / z_{p,-\theta}\right)\left(1 / 2-\beta^{*}\right)\right.}{\left.+\left(z_{p, \theta}^{\prime} / z_{p, \theta}\right)\left(1 / 2+\beta^{*}\right)\right) d \theta}\right)+\mathrm{O}\left(\left|\alpha^{*}+\beta^{*}\right|^{2}\right)\right) \\
= & \mathcal{A}\left(S_{0}^{\prime}, T^{\prime}\right)\left(1+\left(\mathcal{A}_{p, \theta}\left(S^{\prime}, T^{\prime}\right) d \theta\right.\right. \\
& \left.\left.+H_{p, 1 ; S^{\prime}, T^{\prime}}\left(\left\{\beta^{*}\right\}\right)-\beta^{*}\right) \sum_{p}\left(\left.\left.H_{p, 1 ; S^{\prime}, T^{\prime}}\left(\left\{\alpha^{*}\right\}\right)\right|_{\alpha^{*}=-\beta^{*}}\left(\left\{\alpha^{*}\right\}\right)\right|_{\alpha^{*}=-\beta^{*}}-H_{p, 2 ; S^{\prime}, T^{\prime}}\left(\left\{\beta^{*}\right\}\right)\right)\right) \\
+ & O\left(\left|\alpha^{*}+\beta^{*}\right|\right)^{2},
\end{aligned}
$$

where the first line is a result of differentiating (4.2), and the second line from the definitions of $H_{p, 1 ; S, T}(W)$ (in Theorem 4.1) and $H_{p, 2 ; S, T}(W)$ (in (4.24)).

Thus we have

$$
\begin{aligned}
Q_{\zeta ; 1}(S, T) \mathcal{A}(S, T)= & Q_{\zeta}\left(S^{\prime}, T^{\prime}\right) \mathcal{A}\left(S^{\prime}, T^{\prime}\right)\left(1-\left(\alpha^{*}+\beta^{*}\right)\left(-\frac{\chi^{\prime}}{\chi}\left(s-\beta^{*}\right)\right.\right. \\
& \left.\left.+\left.\mathcal{H}_{S^{\prime}, T^{\prime}}\left(\left\{\alpha^{*}\right\}\right)\right|_{\alpha^{*}=-\beta^{*}}+\mathcal{H}_{S^{\prime}, T^{\prime}}\left(\left\{\beta^{*}\right\}\right)\right)\right)+\mathrm{O}(1)
\end{aligned}
$$

as $\alpha^{*} \rightarrow-\beta^{*}$. 
Now we obtain an expansion for the product of $H$ term. By the definition of $H_{\zeta}$ in Theorem 4.1 we have that

$$
\begin{aligned}
H_{\zeta ; S, T}(\{\alpha\}) & =\sum_{\hat{\alpha} \in S} \frac{\zeta^{\prime}}{\zeta}(1+\alpha-\hat{\alpha})-\sum_{\hat{\beta} \in T} \frac{\zeta^{\prime}}{\zeta}(1+\alpha+\hat{\beta}) \\
& =H_{\zeta ; S^{\prime}, T^{\prime}}(\{\alpha\})+\frac{\zeta^{\prime}}{\zeta}\left(1+\alpha-\alpha^{*}\right)-\frac{\zeta^{\prime}}{\zeta}\left(1+\alpha+\beta^{*}\right) ; \\
H_{\zeta ; S, T}(\{\beta\}) & =\sum_{\hat{\beta} \in T} \frac{\zeta^{\prime}}{\zeta}(1+\beta-\hat{\beta})-\sum_{\hat{\alpha} \in S} \frac{\zeta^{\prime}}{\zeta}(1+\beta+\hat{\alpha}) \\
& =H_{\zeta ; S^{\prime}, T^{\prime}}(\{\beta\})+\frac{\zeta^{\prime}}{\zeta}\left(1+\beta-\beta^{*}\right)-\frac{\zeta^{\prime}}{\zeta}\left(1+\beta+\alpha^{*}\right) ;
\end{aligned}
$$

and

$$
H_{\zeta ; S, T}(\{\alpha, \beta\})=\left(\frac{\zeta^{\prime}}{\zeta}\right)^{\prime}(1+\alpha+\beta)=H_{\zeta ; S^{\prime}, T^{\prime}}(\{\alpha, \beta\})
$$

Thus,

$$
\left.H_{\zeta ; S, T}(W)\right|_{\alpha^{*}=-\beta^{*}}=H_{\zeta ; S^{\prime}, T^{\prime}}(W)
$$

and

$$
\begin{aligned}
& \left.\frac{d}{d \alpha^{*}} H_{\zeta ; S, T}(W)\right|_{\alpha^{*}=-\beta^{*}}= \begin{cases}-\left(\frac{\zeta^{\prime}}{\zeta}\right)^{\prime}\left(1+\alpha+\beta^{*}\right) & \text { if } W=\{\alpha\} \subset \bar{S}, \\
-\left(\frac{\zeta^{\prime}}{\zeta}\right)^{\prime}\left(1+\beta-\beta^{*}\right) & \text { if } W=\{\beta\} \subset \bar{T}, \\
0 & \text { otherwise. }\end{cases} \\
& =-\left.H_{\zeta, S^{\prime}, T^{\prime}}\left(W+\left\{\alpha^{*}\right\}\right)\right|_{\alpha^{*}=-\beta^{*}}-H_{\zeta, S^{\prime}, T^{\prime}}\left(W+\left\{\beta^{*}\right\}\right) .
\end{aligned}
$$

In exactly the same way,

$$
\left.H_{p, 1 ; S, T}(W)\right|_{\alpha^{*}=-\beta^{*}}=H_{p, 1 ; S^{\prime}, T^{\prime}}(W)
$$

and

$$
\begin{aligned}
\left.\frac{d}{d \alpha^{*}} H_{p, 1 ; S, T}(W)\right|_{\alpha^{*}=-\beta^{*}}= & -\left.H_{p, 1, S^{\prime}, T^{\prime}}\left(W+\left\{\alpha^{*}\right\}\right)\right|_{\alpha^{*}=-\beta^{*}} \\
& -H_{p, 1, S^{\prime}, T^{\prime}}\left(W+\left\{\beta^{*}\right\}\right) .
\end{aligned}
$$


Also, by Lemma 4.1 we have

$$
\left.H_{p, 2 ; S, T}(W)\right|_{\alpha^{*}=-\beta^{*}}=H_{p, 2 ; S^{\prime}, T^{\prime}}(W)
$$

and

$$
\begin{aligned}
\left.\frac{d}{d \alpha^{*}} H_{p, 2 ; S, T}(W)\right|_{\alpha^{*}=-\beta^{*}}= & -\left.H_{p, 2, S^{\prime}, T^{\prime}}\left(W+\left\{\alpha^{*}\right\}\right)\right|_{\alpha^{*}=-\beta^{*}} \\
& -H_{p, 2, S^{\prime}, T^{\prime}}\left(W+\left\{\beta^{*}\right\}\right) .
\end{aligned}
$$

Combining these results we have exactly equation (4.37).

The rest of the proof proceeds exactly as before.

\section{4. n-Correlation via the Ratios conjecture}

Now we proceed to $n$-correlation. Let $f$ satisfy the conditions

$$
f\left(x_{1}, \ldots, x_{n}\right) \text { is holomorphic for }\left|\Im x_{j}\right|<2 \text {, with } j=1, \ldots, n,
$$

is translation invariant, i.e., $f\left(x_{1}+t, \ldots, x_{n}+t\right)=f\left(x_{1}, \ldots, x_{n}\right)$

$$
\begin{gathered}
\text { and satisfies } f\left(0, x_{2}, \ldots, x_{n}\right) \ll 1 /\left(1+\left|x_{2}\right|^{2}+\cdots+\left|x_{n}\right|^{2}\right) \\
\text { as }\left|x_{j}\right| \rightarrow \infty \text {, with } j=2, \ldots, n .
\end{gathered}
$$

Theorem 4.2. Let $\mathcal{C}_{-}$denote the path from $-\delta+i T$ down to $-\delta-i T$ and let $\mathcal{C}_{+}$denote the path from $\delta-i T$ up to $\delta+i T$ and let $f$ be as in (4.55). Using the notation $J_{\zeta, t}(A ; B ; C)$ from Theorem 4.1,

$$
\begin{aligned}
\sum_{0<\gamma_{j_{1}}, \ldots, \gamma_{j_{n}} \leq T} f\left(\gamma_{j_{1}}, \ldots, \gamma_{j_{n}}\right)= & \frac{1}{(2 \pi i)^{n}} \sum_{K+L+M=\{1, \ldots, n\}}(-1)^{|L|+|M|} \\
& \times \int_{\mathcal{C}_{+}{ }^{K}} \int_{\mathcal{C}_{-}{ }^{L+M}} \frac{1}{T} \int_{I^{*}} J_{\zeta, t}\left(z_{K} ;-z_{L} ;-z_{M}\right) d t \\
& \times f\left(i z_{1}, \ldots, i z_{n}\right) d z_{1} \cdots d z_{n},
\end{aligned}
$$

where $z_{K}=\left\{z_{k}: k \in K\right\},-z_{L}=\left\{-z_{\ell}: \ell \in L\right\}$ and $\int_{\mathcal{C}_{+}{ }^{K}} \int_{\mathcal{C}_{-}{ }^{L+M}}$ means that we are integrating all of the variables in $z_{K}$ along the $\mathcal{C}_{+}$path and all of the variables in $z_{L}$ or $z_{M}$ along the $\mathcal{C}_{-}$path; and $I^{*}$ is the interval which has lower endpoint $\max \left\{0,-\Im z_{1}, \ldots,-\Im z_{n}\right\}$ and upper endpoint $\min \{T, T-$ $\left.\Im z_{1}, \ldots, T-\Im z_{n}\right\}$. 
Proof. By Cauchy's theorem we can express the sum over zeros as

$$
\begin{aligned}
\sum_{0<\gamma_{1}, \ldots, \gamma_{n} \leq T} f\left(\gamma_{1}, \ldots, \gamma_{n}\right)= & \frac{1}{(2 \pi i)^{n}} \int_{\mathcal{C}} \ldots \int_{\mathcal{C}} f\left(-i z_{1}, \ldots,-i z_{n}\right) \\
& \times \prod_{j=1}^{n} \frac{\zeta^{\prime}}{\zeta}\left(1 / 2+z_{j}\right) d z_{1} \ldots d z_{n}
\end{aligned}
$$

where $\mathcal{C}$ is a positively oriented contour which encloses a subinterval of the imaginary axis from zero to $T$. We choose a specific path $\mathcal{C}$ to be the positively oriented rectangle that has vertices $\delta, \delta+i T,-\delta+i T,-\delta$, where $\delta$ is a small positive number.

Due to the translation invariance of $f,(4.57)$ equals

$$
\begin{aligned}
\frac{1}{T} \int_{0}^{T} & \frac{1}{(2 \pi i)^{n}} \int_{\mathcal{C}} \cdots \int_{\mathcal{C}} f\left(-i z_{1}-t, \ldots,-i z_{n}-t\right) \\
& \times \prod_{j=1}^{n} \frac{\zeta^{\prime}}{\zeta}\left(1 / 2+z_{j}\right) d z_{1} \cdots d z_{n} d t \\
= & \frac{1}{T} \int_{0}^{T} \frac{1}{(2 \pi i)^{n}} \int_{\mathcal{C}_{-i t}} \cdots \int_{\mathcal{C}_{-i t}} f\left(-i z_{1}, \ldots,-i z_{n}\right) \\
& \times \prod_{j=1}^{n} \frac{\zeta^{\prime}}{\zeta}\left(1 / 2+i t+z_{j}\right) d z_{1} \cdots d z_{n} d t \\
= & \frac{1}{(2 \pi i)^{n}} \sum_{\epsilon_{j} \in\{-1,+1\}} \int_{\mathcal{C}_{\epsilon_{n}}} \ldots \int_{\mathcal{C}_{\epsilon_{1}}} \frac{1}{T} \int_{I^{*}} f\left(-i z_{1}, \ldots,-i z_{n}\right) \\
& \times \prod_{j=1}^{n} \frac{\zeta^{\prime}}{\zeta}\left(1 / 2+i t+z_{j}\right) d t d z_{1} \cdots d z_{n}+\mathrm{O}\left(T^{\epsilon}\right)
\end{aligned}
$$

where the range of the innermost integral is the interval $I^{*}$ which has lower endpoint $\max \left\{0,-\Im z_{1}, \ldots,-\Im z_{n}\right\}$ and upper endpoint $\min \{T, T-$ $\left.\Im z_{1}, \ldots, T-\Im z_{n}\right\}$. In the second line we made a change of variables $z_{j} \rightarrow$ $z_{j}+i t$. The contour $\mathcal{C}_{-i t}$ is $\mathcal{C}$ shifted down by $-i t$; that is, it runs from $\delta-i t, \delta+i(T-t),-\delta+i(T-t),-\delta-i t$. In progressing to the third line, we note that the horizontal portions of the contour of integration can be chosen so that the integral along them is $\mathrm{O}\left(T^{\epsilon}\right)$ (following the identical argument to Davenport [11, p. 108]), so we concentrate on the vertical sides of the contours. When we now exchange the order of integration to move the $t$ integral to the inside, the integration over $z_{1}, \ldots, z_{n}$ becomes the sum of $2^{n}$ integrals, each on one of the contours $\mathcal{C}_{+}$or $\mathcal{C}_{-}$defined in Theorem 4.2. 
Remark 4.3. The main integral is of size $\approx T \log ^{n} T$. The $T$ is a result of $\int_{[-T, T]^{n}} f \approx T$; the power of the log comes from the moment of the logarithmic derivative and will become clear from the examples at the end of the paper.

For each variable $z_{j}$ in $(4.58)$ which is on $\mathcal{C}_{-}$we use the functional equation

$$
\frac{\zeta^{\prime}}{\zeta}(s)=\frac{\chi^{\prime}}{\chi}(s)-\frac{\zeta^{\prime}}{\zeta}(1-s)
$$

to replace $\frac{\zeta^{\prime}}{\zeta}\left(s+z_{j}\right)$, where $s=1 / 2+i t$. In this way we find that $(4.58)$ equals

$$
\begin{aligned}
& \frac{1}{(2 \pi i)^{n}} \sum_{\epsilon_{j} \in\{-1,+1\}} \int_{\mathcal{C}_{\epsilon_{n}}} \cdots \int_{\mathcal{C}_{\epsilon_{1}}} \frac{1}{T} \int_{I^{*}} \prod_{j=1}^{n}\left(\frac{1-\epsilon_{j}}{2} \frac{\chi^{\prime}}{\chi}\left(s+z_{j}\right)\right. \\
& \left.+\epsilon_{j} \frac{\zeta^{\prime}}{\zeta}\left(1 / 2+\epsilon_{j}\left(i t+z_{j}\right)\right)\right) \times f\left(i z_{1}, \ldots, i z_{n}\right) d t d z_{1} \cdots d z_{n} .
\end{aligned}
$$

Another way to write this equation is

$$
\begin{aligned}
& \frac{1}{(2 \pi i)^{n}} \sum_{K \subset\{1, \ldots, n\}} \prod_{j \in K} \int_{\mathcal{C}_{+}} \prod_{j \notin K} \int_{\mathcal{C}_{-}} \frac{1}{T} \int_{I^{*}} \frac{\zeta^{\prime}}{\zeta}\left(s+z_{j}\right) \\
& \quad \times\left(\frac{\chi^{\prime}}{\chi}\left(s+z_{j}\right)-\frac{\zeta^{\prime}}{\zeta}\left(1-s-z_{j}\right)\right) \times f\left(i z_{1}, \ldots, i z_{n}\right) d t d z_{1} \ldots d z_{n} .
\end{aligned}
$$

(Note that the $d z$ are no longer in order but this should not cause confusion.) The expansion of the product over $j \notin K$ can be easily expressed as a sum over subsets of $K$. This yields

$$
\begin{aligned}
& \frac{1}{(2 \pi i)^{n}} \sum_{K+L+M=\{1, \ldots, n\}}(-1)^{|L|+|M|} \prod_{k \in K} \int_{\mathcal{C}_{+}} \prod_{\ell \in L} \int_{\mathcal{C}_{-}} \frac{1}{T} \int_{I^{*}} \frac{\zeta^{\prime}}{\zeta}\left(s+z_{k}\right) \\
& \times \frac{\zeta^{\prime}}{\zeta}\left(1-s-z_{\ell}\right) \prod_{m \in M} \int_{\mathcal{C}_{-}}\left(-\frac{\chi^{\prime}}{\chi}\left(s+z_{m}\right)\right) f\left(i z_{1}, \ldots, i z_{n}\right) d t d z_{1} \ldots d z_{n} .
\end{aligned}
$$


Remark 4.4. We note the asymptotic for $\frac{\chi^{\prime}}{\chi}$ :

$$
\frac{\chi^{\prime}}{\chi}(1 / 2+i t)=-\log \frac{|t|}{2 \pi}\left(1+\mathrm{O}\left(\frac{1}{|t|}\right)\right) \text {. }
$$

In some applications $\left|z_{m}\right|$ is small relative to $t$, and it simplifies the formulae to replace $\frac{\chi^{\prime}}{\chi}\left(s+z_{m}\right)$ with $-\log \frac{|t|}{2 \pi}$. However, here where $z_{k}$ can be the same size as $t$ we will not use this approximation.

We have the statement of Theorem 4.2.

\section{5. $n$-Correlation for the Riemann zeros}

We will now state our main theorem.

Theorem 4.3. Assume the Ratios conjecture 4.1. Let $J_{\zeta, t}^{*}$ be as defined in Theorem 4.1. Then

$$
\begin{aligned}
& \sum_{0<\gamma_{1} \neq \cdots \neq \gamma_{n} \leq T} f\left(\gamma_{1}, \ldots, \gamma_{n}\right) \\
= & \frac{1}{(2 \pi)^{n}} \int_{[-T, T]^{n}} \frac{1}{T} \int_{I^{*}} \sum_{K+L+M=\{1, \ldots, n\}} J_{\zeta, t}^{*}\left(-i z_{K} ; i z_{L} ; i z_{M}\right) d t \\
& \times f\left(z_{1}, \ldots, z_{n}\right) d z_{1} \ldots d z_{n}+\mathrm{O}\left(T^{1 / 2+\epsilon}\right),
\end{aligned}
$$

where $-i z_{K}=\left\{-i z_{k}: k \in K\right\} i z_{L}=\left\{i z_{\ell}: \ell \in L\right\}$ and $i z_{M}=\left\{i z_{m}: m \in M\right\}$. Moreover, the integrand has no poles on the path of integration.

The proof is nearly identical to that of Theorem 3.5. The only difference is that some care is needed with regard to endpoints of intervals when we move each new path of integration onto the imaginary axis. The (slight) difficulty is with poles that may lie at the very endpoints; this point did not arise in the random matrix theory context because of the periodicity of the integrand. However by extending the paths slightly we can circumvent this difficulty; an argument like that used to handle the horizontal segments in the proof of Theorem 4.2 will work in this case too, and introduces an error term of size only $\mathrm{O}\left(T^{\epsilon}\right)$.

It remains to verify that the integrand in Theorem 4.3 has no poles on the path of integration. We have already confirmed in Lemma 3.2 that each $J^{*}\left(-i \theta_{K} ; i \theta_{L}\right)$ has only a simple pole at $\theta_{k}=-\theta_{\ell}$ for $\theta_{k} \in \theta_{K}$ and $\theta_{\ell} \in \theta_{L}$. 
We check that

$$
\sum_{K+L+M=\{1,2, \ldots, n\}} J_{\zeta, t}^{*}\left(-i \theta_{K} ; i \theta_{L} ; i \theta_{M}\right)
$$

has no pole at $\theta_{1}=\theta_{2}$ when the values of the remaining $\theta_{j}$ are not equal to $\theta_{1}$ or $\theta_{2}$. A given $J_{\zeta, t}^{*}\left(-i \theta_{K} ; i \theta_{L} ; i \theta_{M}\right)$ only has a pole when $\theta_{1} \in \theta_{L}$ and $\theta_{2} \in \theta_{K}$, or vice versa, so

$$
\begin{aligned}
\underset{\theta_{1}=\theta_{2}}{\operatorname{Res}} \sum_{K+L+M=\{1,2, \ldots, n\}} J_{\zeta, t}^{*}\left(-i \theta_{K} ; i \theta_{L} ; i \theta_{M}\right) \\
=\sum_{K+L+M=\{3, \ldots, n\}} \operatorname{Res}_{\theta_{1}=\theta_{2}}^{\operatorname{Res}}\left(J_{\zeta, t}^{*}\left(-i \theta_{K}+\left\{-i \theta_{1}\right\} ;\left\{i \theta_{2}\right\}+i \theta_{L} ; i \theta_{M}\right)\right. \\
\left.\quad+J_{\zeta, t}^{*}\left(-i \theta_{K}+\left\{-i \theta_{2}\right\} ;\left\{i \theta_{1}\right\}+i \theta_{L} ; i \theta_{M}\right)\right)=0
\end{aligned}
$$

this is zero because $\operatorname{Res}_{s=x} f(s, x)=-\operatorname{Res}_{s=x} f(x, s)$.

Thus if (4.65) had a singular set it would be of complex dimension less than $n-1$ and by standard results in the theory of several complex variables, this implies that there is no singular set (see for example [16, Corollary 7.3.2]).

Our proof of $n$-correlation in the case of $\zeta$-zeros is now complete.

Corollary 4.2. By rearranging the integrals, now that we know the integrand has no singularities, and using the fact that $f$ is translation invariant we have that the Ratios conjecture implies that

$$
\begin{aligned}
& \sum_{0<\gamma_{1} \neq \cdots \neq \gamma_{n} \leq T} f\left(\gamma_{1}, \ldots, \gamma_{n}\right) \\
= & \frac{1}{T} \int_{0}^{T} \frac{1}{(2 \pi)^{n}} \int_{[-T, T]^{n}} \sum_{K+L+M=\{1, \ldots, n\}} J_{\zeta, t}^{*}\left(-i z_{K}+i t ; i z_{L}-i t ; i z_{M}-i t\right) \\
& \times f\left(z_{1}, \ldots, z_{n}\right) d z_{1} \cdots d z_{n} d t+O\left(T^{1 / 2+\epsilon}\right)
\end{aligned}
$$

\section{Examples}

In this section we explicitly write out all of the terms in our expressions for $n$-correlations for RMT eigenvalues and for $\zeta$-zeros for $2 \leq n \leq 4$. In the case of the $\zeta$ correlations, we simplify the terms $X_{t}(S, T)$ and $\frac{\chi^{\prime}}{\chi}(s+\alpha)$ using the approximations involving $\ell=\log \frac{t}{2 \pi}$ mentioned at (4.8) and (4.63). 
To proceed, we calculate $J^{*}(A ; B)$ and $J_{\zeta, t}^{*}(A ; B):=J_{\zeta, t}^{*}(A ; B ; U) /$ $\prod_{\mu \in U} \frac{\chi^{\prime}}{\chi}\left(\frac{1}{2}+i t+\mu\right)$ for sets $A$ and $B$ with four or fewer elements. $D_{S, T}$ and $D_{\zeta, S, T}$ are defined in the proofs of Lemmas 3.2 and 4.2, respectively. In the following sections we first compile $D_{S, T}, D_{\zeta, S, T}$, then evaluate $J^{*}$ and $J_{\zeta, t}^{*}$ and then assemble these into the correlation formulas $R_{N, n}\left(x_{1}, \ldots, x_{n}\right)$ and $R_{\zeta, t, n}\left(x_{1}, \ldots, x_{n}\right)$. Here we define $R$ via

$$
\begin{aligned}
& \int_{U(N)_{1 \leq j_{1}, \ldots, j_{n} \leq N}} \sum^{*} f\left(\theta_{1}, \ldots, \theta_{n}\right) d X_{N} \\
& \quad=\frac{1}{(2 \pi)^{n}} \int_{[0,2 \pi]^{n}} R_{N, n}\left(x_{1}, \ldots, x_{n}\right) f\left(x_{1}, \ldots, x_{n}\right) d x_{1} \cdots d x_{n}
\end{aligned}
$$

and

$$
\sum_{0<\gamma_{1} \neq \cdots \neq \gamma_{n} \leq T} f\left(\gamma_{1}, \ldots, \gamma_{n}\right)=\frac{1}{(2 \pi)^{n}} \int_{[-T, T]^{n}} \frac{1}{T} \int_{I^{*}} R_{\zeta, t, n}\left(x_{1}, \ldots, x_{n}\right) d t
$$

$$
\times f\left(x_{1}, \ldots, x_{n}\right) d x_{1} \cdots d x_{n}+\mathrm{O}\left(T^{1 / 2+\epsilon}\right),
$$

(compare these with Theorems 3.5 and 4.3).

Recall that

$$
\begin{aligned}
z(x) & =\frac{1}{\left(1-e^{-x}\right)}, \\
S(x) & =S_{N}(x)=\frac{\sin (N x / 2)}{\sin (x / 2)} \\
z_{p}(x) & :=\left(1-p^{-x}\right)^{-1}
\end{aligned}
$$

and

$$
Z_{p}(A, B)=\prod_{\substack{\alpha \in A \\ \beta \in B}} z_{p}(1+\alpha+\beta)^{-1} .
$$

We also will introduce, as needed below, a number of other expressions $A(x), B(x)$, etc.; for convenience, these are listed in Section 5.7.

\subsection{Pair correlation, RMT}

Suppose that the sets $A$ and $B$ have just one element: $A=\{a\}, B=\{b\}$. We have

$$
J^{*}(a ; b)=D_{\phi, \phi}+D_{a, b},
$$


where

$$
D_{\phi, \phi}=\left(\frac{z^{\prime}}{z}\right)^{\prime}(a+b)
$$

and

$$
D_{a, b}=e^{-N(a+b)} z(a+b) z(-a-b) .
$$

Thus

$$
J^{*}(a ; b)=\left(\frac{z^{\prime}}{z}\right)^{\prime}(a+b)+e^{-N(a+b)} z(a+b) z(-a-b) .
$$

Then the 2-point correlation function is

$$
\begin{aligned}
R_{N, 2}(u, v) & =N^{2}+J^{*}(i u ;-i v)+J^{*}(-i u ; i v) \\
& =\operatorname{det}\left(\begin{array}{cc}
N & S(u-v) \\
S(v-u) & N
\end{array}\right) .
\end{aligned}
$$

\subsection{Pair correlation, $\zeta$}

We have

$$
\begin{aligned}
J_{\zeta, t}^{*}(a ; b)= & \left(\frac{\zeta^{\prime}}{\zeta}\right)^{\prime}(1+a+b)-B(a+b) \\
& +e^{-\ell(a+b)} \zeta(1+a+b) \zeta(1-a-b) A(a+b),
\end{aligned}
$$

where $\ell=\log \frac{t}{2 \pi}$ and

$$
\begin{aligned}
& A(x)=\prod_{p} \frac{\left(1-1 / p^{1+x}\right)\left(1-2 / p+1 / p^{1+x}\right)}{(1-1 / p)^{2}}, \\
& B(x)=\sum_{p}\left(\frac{\log p}{p^{1+x}-1}\right)^{2},
\end{aligned}
$$

and conjecture that

$$
R_{\zeta, t, 2}(u, v)=\ell^{2}+J_{\zeta}^{*}(i u ;-i v)+J_{\zeta}^{*}(-i u ; i v) .
$$

Further, letting

$$
P_{1}(x)=e^{-\ell x} A(x) \zeta(1+x) \zeta(1-x)
$$


and

$$
P_{2}(x)=\left(\frac{\zeta^{\prime}}{\zeta}\right)^{\prime}(1+x)-B(x)
$$

we have

$$
J_{\zeta, t}^{*}(a ; b)=P_{1}(a+b)+P_{2}(a+b) .
$$

\subsection{Triple correlation, RMT}

In this case we have

$$
\begin{aligned}
J^{*}\left(a ; b_{1}, b_{2}\right) & =D_{\phi, \phi}+D_{a, b_{1}}+D_{a, b_{2}}, \\
(5.20) \quad D_{\phi, \phi} & =0, \\
(5.21) \quad D_{a, b_{1}} & =e^{-N\left(a+b_{1}\right)} z\left(a+b_{1}\right) z\left(-a-b_{1}\right)\left(\frac{z^{\prime}}{z}\left(b_{2}-b_{1}\right)-\frac{z^{\prime}}{z}\left(b_{2}+a\right)\right),
\end{aligned}
$$

and

$$
D_{a, b_{2}}=e^{-N\left(a+b_{2}\right)} z\left(a+b_{2}\right) z\left(-a-b_{2}\right)\left(\frac{z^{\prime}}{z}\left(b_{1}-b_{2}\right)-\frac{z^{\prime}}{z}\left(b_{1}+a\right)\right)
$$

Thus,

$$
\begin{aligned}
J^{*}\left(a ; b_{1}, b_{2}\right)= & e^{-N\left(a+b_{1}\right)} z\left(a+b_{1}\right) z\left(-a-b_{1}\right)\left(\frac{z^{\prime}}{z}\left(b_{2}-b_{1}\right)-\frac{z^{\prime}}{z}\left(b_{2}+a\right)\right) \\
& +e^{-N\left(a+b_{2}\right)} z\left(a+b_{2}\right) z\left(-a-b_{2}\right)\left(\frac{z^{\prime}}{z}\left(b_{1}-b_{2}\right)-\frac{z^{\prime}}{z}\left(b_{1}+a\right)\right) .
\end{aligned}
$$

Then

$$
\begin{aligned}
R_{N, 3}(u, v, w)= & N^{3}+N\left(J^{*}(i u ;-i v)+J^{*}(i v ;-i u)+J^{*}(i u ;-i w)\right. \\
& \left.+J^{*}(i w ;-i u)+J^{*}(i w ;-i v)+J^{*}(i v ;-i w)\right) \\
& +\left(J^{*}(-i w ; i u, i v)+J^{*}(-i v ; i w, i u)+J^{*}(-i u ; i w, i v)\right. \\
& \left.+J^{*}(i u ;-i w,-i v)+J^{*}(i v ;-i w,-i u)+J^{*}(i w ;-i u,-i v)\right) \\
(5.24) \quad & \operatorname{det}\left(\begin{array}{ccc}
N & S(u-v) & S(u-w) \\
S(v-u) & N & S(v-w)
\end{array}\right) .
\end{aligned}
$$




\subsection{Triple correlation for $\zeta$}

The analog for $\zeta$ is

$$
\begin{aligned}
J_{\zeta, t}^{*}\left(a ; b_{1}, b_{2}\right)= & e^{-\ell\left(a+b_{1}\right)} A\left(a+b_{1}\right) \zeta\left(1+a+b_{1}\right) \zeta\left(1-a-b_{1}\right) \\
& \times\left(\frac{\zeta^{\prime}}{\zeta}\left(1+b_{2}-b_{1}\right)-\frac{\zeta^{\prime}}{\zeta}\left(1+a+b_{2}\right)-B_{1}\left(a+b_{1}, a+b_{2}\right)\right) \\
& +e^{-\ell\left(a+b_{2}\right)} A\left(a+b_{2}\right) \zeta\left(1+a+b_{2}\right) \zeta\left(1-a-b_{2}\right) \\
& \times\left(\frac{\zeta^{\prime}}{\zeta}\left(1+b_{1}-b_{2}\right)-\frac{\zeta^{\prime}}{\zeta}\left(1+a+b_{1}\right)-B_{1}\left(a+b_{2}, a+b_{1}\right)\right) \\
& +Q\left(a+b_{1}, a+b_{2}\right),
\end{aligned}
$$

where

$$
Q(x, y)=-\sum_{p} \frac{\log ^{3} p}{p^{2+x+y}\left(1-1 / p^{1+x}\right)\left(1-1 / p^{1+y}\right)}
$$

and

$$
B_{1}(x, y)=\sum_{p} \frac{\left(1-1 / p^{x}\right)\left(1-1 / p^{x}-1 / p^{y}+1 / p^{1+y}\right) \log p}{\left(1-1 / p^{1-x+y}\right)\left(1-1 / p^{1+y}\right)\left(1-2 / p+1 / p^{1+x}\right) p^{2-x+y}} .
$$

Then we conjecture that

$$
\begin{aligned}
R_{\zeta, t, 3}(u, v, w)= & \ell^{3}+\ell\left(J_{\zeta, t}^{*}(i u ;-i v)+J_{\zeta, t}^{*}(i v ;-i u)+J_{\zeta, t}^{*}(i u ;-i w)\right. \\
& \left.+J_{\zeta, t}^{*}(i w ;-i u)+J_{\zeta, t}^{*}(i w ;-i v)+J_{\zeta, t}^{*}(i v ;-i w)\right) \\
& +\left(J_{\zeta, t}^{*}(-i w ; i u, i v)+J_{\zeta, t}^{*}(-i v ; i w, i u)+J_{\zeta, t}^{*}(-i u ; i w, i v)\right. \\
& +J_{\zeta, t}^{*}(i u ;-i w,-i v)+J_{\zeta, t}^{*}(i v ;-i w,-i u)+J_{\zeta, t}^{*}(i w ;-i u,-i v) .
\end{aligned}
$$

It is convenient to introduce the function

$$
P_{3}(a, b, c)=B_{1}(a+b, a+c)+\frac{\zeta^{\prime}}{\zeta}(1+a+c)-\frac{\zeta^{\prime}}{\zeta}(1+c-b)
$$

In terms of this, we have

$$
\begin{aligned}
J_{\zeta, t}^{*}\left(\{a\} ;\left\{b_{1}, b_{2}\right\}\right)= & Q\left(a+b_{1}, a+b_{2}\right)-P_{1}\left(a+b_{1}\right) P_{3}\left(a, b_{1}, b_{2}\right) \\
& -P_{1}\left(a+b_{2}\right) P_{3}\left(a, b_{2}, b_{1}\right) .
\end{aligned}
$$




\subsection{Quadruple correlation, RMT}

With $A=\{a\}, B=\left\{b_{1}, b_{2}, b_{3}\right\}$, we have that

$$
\begin{aligned}
J^{*}\left(a ; b_{1}, b_{2}, b_{3}\right)= & D_{\phi, \phi}+D_{a, b_{1}}+D_{a, b_{2}}+D_{a, b_{3}} \\
= & e^{-N\left(a+b_{1}\right)} z\left(a+b_{1}\right) z\left(-a-b_{1}\right)\left(\frac{z^{\prime}}{z}\left(b_{2}-b_{1}\right)-\frac{z^{\prime}}{z}\left(b_{2}+a\right)\right) \\
& \times\left(\frac{z^{\prime}}{z}\left(b_{3}-b_{1}\right)-\frac{z^{\prime}}{z}\left(b_{3}+a\right)\right) \\
& +e^{-N\left(a+b_{2}\right)} z\left(a+b_{2}\right) z\left(-a-b_{2}\right)\left(\frac{z^{\prime}}{z}\left(b_{1}-b_{2}\right)-\frac{z^{\prime}}{z}\left(b_{1}+a\right)\right) \\
& \times\left(\frac{z^{\prime}}{z}\left(b_{3}-b_{2}\right)-\frac{z^{\prime}}{z}\left(b_{3}+a\right)\right) \\
& +e^{-N\left(a+b_{3}\right)} z\left(a+b_{3}\right) z\left(-a-b_{3}\right)\left(\frac{z^{\prime}}{z}\left(b_{1}-b_{3}\right)-\frac{z^{\prime}}{z}\left(b_{1}+a\right)\right) \\
& \times\left(\frac{z^{\prime}}{z}\left(b_{2}-b_{3}\right)-\frac{z^{\prime}}{z}\left(b_{2}+a\right)\right) .
\end{aligned}
$$

With $A=\left\{a_{1}, a_{2}\right\}, B=\left\{b_{1}, b_{2}\right\}$, we have

$$
J^{*}\left(a_{1}, a_{2} ; b_{1}, b_{2}\right)=D_{\phi, \phi}+D_{a_{1}, b_{1}}+D_{a_{1}, b_{2}}+D_{a_{2}, b_{1}}+D_{a_{2}, b_{2}}+D_{a_{1}, a_{2}, b_{1}, b_{2}},
$$

where

$$
D_{\phi, \phi}=\left(\frac{z^{\prime}}{z}\right)^{\prime}\left(a_{1}+b_{1}\right)\left(\frac{z^{\prime}}{z}\right)^{\prime}\left(a_{2}+b_{2}\right)+\left(\frac{z^{\prime}}{z}\right)^{\prime}\left(a_{1}+b_{2}\right)\left(\frac{z^{\prime}}{z}\right)^{\prime}\left(a_{2}+b_{1}\right)
$$

and

$$
\begin{aligned}
D_{a_{1}, b_{1}}= & e^{-N\left(a_{1}+b_{1}\right)} z\left(a_{1}+b_{1}\right) z\left(-a_{1}-b_{1}\right) \\
& \times\left(H_{\left\{a_{1}\right\},\left\{b_{1}\right\}}\left(\left\{a_{2}\right\},\left\{b_{2}\right\}\right)+H_{\left\{a_{1}\right\},\left\{b_{1}\right\}}\left(\left\{a_{2}\right\}\right) H_{\left\{a_{1}\right\},\left\{b_{1}\right\}}\left(\left\{b_{2}\right\}\right)\right) \\
= & e^{-N\left(a_{1}+b_{1}\right)} z\left(a_{1}+b_{1}\right) z\left(-a_{1}-b_{1}\right)\left(\left(\frac{z^{\prime}}{z}\right)^{\prime}\left(a_{2}+b_{2}\right)\right. \\
& \left.+\left(\frac{z^{\prime}}{z}\left(a_{2}-a_{1}\right)-\frac{z^{\prime}}{z}\left(a_{2}+b_{1}\right)\right)\left(\frac{z^{\prime}}{z}\left(b_{2}-b_{1}\right)-\frac{z^{\prime}}{z}\left(b_{2}+a_{1}\right)\right)\right) ;
\end{aligned}
$$


the other $D_{a_{i}, b_{j}}$ are similar. Also,

$$
\begin{aligned}
D_{a_{1}, a_{2}, b_{1}, b_{2}}= & e^{-N\left(a_{1}+a_{2}+b_{1}+b_{2}\right)} \\
& \times \frac{z\left(a_{1}+b_{1}\right) z\left(-a_{1}-b_{1}\right) z\left(a_{1}+b_{2}\right) z\left(-a_{1}-b_{2}\right) z\left(a_{2}+b_{1}\right)}{z\left(a_{1}-a_{2}\right) z\left(a_{2}-a_{1}\right) z\left(b_{1}-b_{2}\right) z\left(b_{2}-b_{1}\right)} .
\end{aligned}
$$

Thus,

$$
\begin{aligned}
& J^{*}\left(a_{1}, a_{2} ; b_{1}, b_{2}\right) \\
& :=\left(\frac{z^{\prime}}{z}\right)^{\prime}\left(a_{1}+b_{1}\right)\left(\frac{z^{\prime}}{z}\right)^{\prime}\left(a_{2}+b_{2}\right)+\left(\frac{z^{\prime}}{z}\right)^{\prime}\left(a_{1}+b_{2}\right)\left(\frac{z^{\prime}}{z}\right)^{\prime}\left(a_{2}+b_{1}\right) \\
& +e^{-N\left(a_{1}+b_{1}\right)} z\left(a_{1}+b_{1}\right) z\left(-a_{1}-b_{1}\right) \\
& \times\left(\left(\frac{z^{\prime}}{z}\right)^{\prime}\left(a_{2}+b_{2}\right)+\left(\frac{z^{\prime}}{z}\left(a_{2}-a_{1}\right)-\frac{z^{\prime}}{z}\left(a_{2}+b_{1}\right)\right)\right. \\
& \left.\times\left(\frac{z^{\prime}}{z}\left(b_{2}-b_{1}\right)-\frac{z^{\prime}}{z}\left(b_{2}+a_{1}\right)\right)\right)+e^{-N\left(a_{1}+b_{2}\right)} z\left(a_{1}+b_{2}\right) z\left(-a_{1}-b_{2}\right) \\
& \times\left(\left(\frac{z^{\prime}}{z}\right)^{\prime}\left(a_{2}+b_{1}\right)+\left(\frac{z^{\prime}}{z}\left(a_{2}-a_{1}\right)-\frac{z^{\prime}}{z}\left(a_{2}+b_{2}\right)\right)\right. \\
& \left.\times\left(\frac{z^{\prime}}{z}\left(b_{1}-b_{2}\right)-\frac{z^{\prime}}{z}\left(b_{1}+a_{1}\right)\right)\right)+e^{-N\left(a_{2}+b_{1}\right)} z\left(a_{2}+b_{1}\right) z\left(-a_{2}-b_{1}\right) \\
& \times\left(\left(\frac{z^{\prime}}{z}\right)^{\prime}\left(a_{1}+b_{2}\right)+\left(\frac{z^{\prime}}{z}\left(a_{1}-a_{2}\right)-\frac{z^{\prime}}{z}\left(a_{1}+b_{1}\right)\right)\right. \\
& \left.\times\left(\frac{z^{\prime}}{z}\left(b_{2}-b_{1}\right)-\frac{z^{\prime}}{z}\left(b_{2}+a_{2}\right)\right)\right)+e^{-N\left(a_{2}+b_{2}\right)} z\left(a_{2}+b_{2}\right) z\left(-a_{2}-b_{2}\right) \\
& \times\left(\left(\frac{z^{\prime}}{z}\right)^{\prime}\left(a_{1}+b_{1}\right)+\left(\frac{z^{\prime}}{z}\left(a_{1}-a_{2}\right)-\frac{z^{\prime}}{z}\left(a_{1}+b_{2}\right)\right)\right. \\
& \left.\times\left(\frac{z^{\prime}}{z}\left(b_{1}-b_{2}\right)-\frac{z^{\prime}}{z}\left(b_{1}+a_{2}\right)\right)\right) \\
& +e^{-N\left(a_{1}+a_{2}+b_{1}+b_{2}\right)} z\left(a_{1}+b_{1}\right) z\left(a_{1}+b_{2}\right) z\left(a_{2}+b_{1}\right) z\left(a_{2}+b_{2}\right) \\
& \times \frac{z\left(-a_{1}-b_{1}\right) z\left(-a_{1}-b_{2}\right) z\left(-a_{2}-b_{1}\right) z\left(-a_{2}-b_{2}\right)}{z\left(a_{1}-a_{2}\right) z\left(a_{2}-a_{1}\right) z\left(b_{1}-b_{2}\right) z\left(b_{2}-b_{1}\right)} .
\end{aligned}
$$


Then

$$
\begin{aligned}
& R_{N, 4}(u, v, w, y) \\
& =N^{4}+N^{2}\left(J^{*}(i u ;-i v)+J^{*}(i v ;-i u)+J^{*}(i u ;-i w)\right. \\
& +J^{*}(i w ;-i u)+J^{*}(i w ;-i v)+J^{*}(i v ;-i w) \\
& +J^{*}(i y ;-i u)+J^{*}(i u ;-i y)+J^{*}(i y ;-i v) \\
& \left.+J^{*}(i v ;-i y)+J^{*}(i y ;-i w)+J^{*}(i w ;-i y)\right) \\
& +N\left(J^{*}(-i w ; i u, i v)+J^{*}(-i v ; i w, i u)+J^{*}(-i u ; i w, i v)\right. \\
& +J^{*}(i u ;-i w,-i v)+J^{*}(i v ;-i w,-i u)+J^{*}(i w ;-i u,-i v) \\
& +J^{*}(-i w ; i y, i v)+J^{*}(-i v ; i w, i y)+J^{*}(-i y ; i w, i v) \\
& +J^{*}(i y ;-i w,-i v)+J^{*}(i v ;-i w,-i y)+J^{*}(i w ;-i y,-i v) \\
& +J^{*}(-i w ; i u, i y)+J^{*}(-i y ; i w, i u)+J^{*}(-i u ; i w, i y) \\
& +J^{*}(i u ;-i w,-i y)+J^{*}(i y ;-i w,-i u)+J^{*}(i w ;-i u,-i y) \\
& +J^{*}(-i y ; i u, i v)+J^{*}(-i v ; i y, i u)+J^{*}(-i u ; i y, i v) \\
& \left.+J^{*}(i u ;-i y,-i v)+J^{*}(i v ;-i y,-i u)+J^{*}(i y ;-i u,-i v)\right) \\
& +J^{*}(-i y ; i u, i v, i w)+J^{*}(-i w ; i y, i u, i v)+J^{*}(-i v ; i y, i u, i w) \\
& +J^{*}(-i u ; i y, i v, i w)+J^{*}(i y ;-i u,-i v,-i w)+J^{*}(i u ;-i y,-i v,-i w) \\
& +J^{*}(i v ;-i y,-i u,-i w)+J^{*}(i w ;-i y,-i u,-i v) \\
& +J^{*}(i y, i u ;-i v,-i w)+J^{*}(i y, i v ;-i u,-i w)+J^{*}(i y, i w ;-i u,-i v) \\
& +J^{*}(i u, i v ;-i y,-i w)+J^{*}(i u, i w ;-i y,-i v)+J^{*}(i v, i w ;-i y,-i u) \\
& =\operatorname{det}\left(\begin{array}{cccc}
N & S(u-v) & S(u-w) & S(u-y) \\
S(v-u) & N & S(v-w) & S(v-y) \\
S(w-u) & S(w-v) & N & S(w-y) \\
S(y-u) & S(y-v) & S(y-w) & N
\end{array}\right) .
\end{aligned}
$$

\subsection{Quadruple correlation, $\zeta$}

We conjecture that

$$
\begin{aligned}
R_{\zeta, t, 4} & (u, v, w, y) \\
= & \ell^{4}+\ell^{2}\left(J_{\zeta, t}^{*}(i u ;-i v)+J_{\zeta, t}^{*}(i v ;-i u)+J_{\zeta, t}^{*}(i u ;-i w)\right. \\
& +J_{\zeta, t}^{*}(i w ;-i u)+J_{\zeta, t}^{*}(i w ;-i v)+J_{\zeta, t}^{*}(i v ;-i w) \\
& +J_{\zeta, t}^{*}(i y ;-i u)+J_{\zeta, t}^{*}(i u ;-i y)+J_{\zeta, t}^{*}(i y ;-i v)
\end{aligned}
$$




$$
\begin{aligned}
& \left.+J_{\zeta, t}^{*}(i v ;-i y)+J_{\zeta, t}^{*}(i y ;-i w)+J_{\zeta, t}^{*}(i w ;-i y)\right) \\
& +\ell\left(J_{\zeta, t}^{*}(-i w ; i u, i v)+J_{\zeta, t}^{*}(-i v ; i w, i u)+J_{\zeta, t}^{*}(-i u ; i w, i v)\right. \\
& +J_{\zeta, t}^{*}(i u ;-i w,-i v)+J_{\zeta, t}^{*}(i v ;-i w,-i u)+J_{\zeta, t}^{*}(i w ;-i u,-i v) \\
& +J_{\zeta, t}^{*}(-i w ; i y, i v)+J_{\zeta, t}^{*}(-i v ; i w, i y)+J_{\zeta, t}^{*}(-i y ; i w, i v) \\
& +J_{\zeta, t}^{*}(i y ;-i w,-i v)+J_{\zeta, t}^{*}(i v ;-i w,-i y)+J_{\zeta, t}^{*}(i w ;-i y,-i v) \\
& +J_{\zeta, t}^{*}(-i w ; i u, i y)+J_{\zeta, t}^{*}(-i y ; i w, i u)+J_{\zeta, t}^{*}(-i u ; i w, i y) \\
& +J_{\zeta, t}^{*}(i u ;-i w,-i y)+J_{\zeta, t}^{*}(i y ;-i w,-i u)+J_{\zeta, t}^{*}(i w ;-i u,-i y) \\
& +J_{\zeta, t}^{*}(-i y ; i u, i v)+J_{\zeta, t}^{*}(-i v ; i y, i u)+J_{\zeta, t}^{*}(-i u ; i y, i v) \\
& \left.+J_{\zeta, t}^{*}(i u ;-i y,-i v)+J_{\zeta, t}^{*}(i v ;-i y,-i u)+J_{\zeta, t}^{*}(i y ;-i u,-i v)\right) \\
& +J_{\zeta, t}^{*}(-i y ; i u, i v, i w)+J_{\zeta, t}^{*}(-i w ; i y, i u, i v)+J_{\zeta, t}^{*}(-i v ; i y, i u, i w) \\
& +J_{\zeta, t}^{*}(-i u ; i y, i v, i w)+J_{\zeta, t}^{*}(i y ;-i u,-i v,-i w)+J_{\zeta, t}^{*}(i u ;-i y,-i v,-i w) \\
& +J_{\zeta, t}^{*}(i v ;-i y,-i u,-i w)+J_{\zeta, t}^{*}(i w ;-i y,-i u,-i v) \\
& +J_{\zeta, t}^{*}(i y, i u ;-i v,-i w)+J_{\zeta, t}^{*}(i y, i v ;-i u,-i w)+J_{\zeta, t}^{*}(i y, i w ;-i u,-i v) \\
& +J_{\zeta, t}^{*}(i u, i v ;-i y,-i w)+J_{\zeta, t}^{*}(i u, i w ;-i y,-i v)+J_{\zeta, t}^{*}(i v, i w ;-i y,-i u),
\end{aligned}
$$

where the relevant $J_{\zeta, t}^{*}$ are now described.

We have

$$
\begin{aligned}
J_{\zeta, t}^{*}\left(\{a\},\left\{b_{1}, b_{2}, b_{3}\right\}\right) \\
=-\sum_{p} \frac{z_{p}\left(1+a+b_{1}\right) z_{p}\left(1+a+b_{2}\right) z_{p}\left(1+a+b_{3}\right) \log ^{4} p}{p^{3+3 a+b_{1}+b_{2}+b_{3}}} \\
\quad+W_{1}\left(a, b_{1} ; b_{2}, b_{3}\right)+W_{1}\left(a, b_{2} ; b_{1}, b_{3}\right)+W_{1}\left(a, b_{3} ; b_{1}, b_{2}\right),
\end{aligned}
$$

where

$$
W_{1}\left(a, b_{1} ; b_{2}, b_{3}\right)=P_{1}\left(a+b_{1}\right)\left(P_{3}\left(a, b_{1}, b_{2}\right) P_{3}\left(a, b_{1}, b_{3}\right)-B_{2}\left(a, b_{1} ; b_{2}, b_{3}\right)\right)
$$

with

$$
B_{2}\left(a, b_{1} ; b_{2}, b_{3}\right)=\sum_{p} \frac{(p-1) p^{2 b_{1}}\left(p^{a+b_{1}}-1\right)\left(p^{a+b_{1}}-p\right) \log ^{2} p}{\left(-2 p^{a+b_{1}}+p^{a+b_{1}+1}+1\right)^{2}\left(p^{b_{1}}-p^{b_{2}+1}\right)\left(p^{b_{1}}-p^{b_{3}+1}\right)} .
$$


We also have

$$
\begin{aligned}
& J_{\zeta, t}^{*}\left(\left\{a_{1}, a_{2}\right\},\left\{b_{1}, b_{2}\right\}\right) \\
& =P_{2}\left(a_{1}+b_{1}\right) P_{2}\left(a_{2}+b_{2}\right)+P_{2}\left(a_{1}+b_{2}\right) P_{2}\left(a_{2}+b_{1}\right)-B_{4}\left(a_{1}, a_{2}, b_{1}, b_{2}\right) \\
& \quad+e^{-\ell\left(a_{1}+a_{2}+b_{1}+b_{2}\right)} A^{*}\left(a_{1}, a_{2}, b_{1}, b_{2}\right) \\
& \quad \times \frac{Z_{\zeta}\left(\left\{a_{1}, a_{2}\right\},\left\{b_{1}, b_{2}\right\}\right) Z_{\zeta}\left(\left\{-a_{1},-a_{2}\right\},\left\{-b_{1},-b_{2}\right\}\right)}{Z_{\zeta}^{\dagger}\left(\left\{a_{1}, a_{2}\right\},\left\{-a_{1},-a_{2}\right\}\right) Z_{\zeta}^{\dagger}\left(\left\{b_{1}, b_{2}\right\},\left\{-b_{1},-b_{2}\right\}\right)}+W\left(a_{1}, b_{1} ; a_{2}, b_{2}\right) \\
& \quad+W\left(a_{1}, b_{2} ; a_{2}, b_{1}\right)+W\left(a_{2}, b_{1} ; a_{1}, b_{2}\right)+W\left(a_{2}, b_{2} ; a_{1}, b_{1}\right),
\end{aligned}
$$

where

$$
\begin{aligned}
A^{*} & \left(a_{1}, a_{2}, b_{1}, b_{2}\right) \\
= & \prod_{p} \frac{Z_{p}\left(\left\{a_{1}, a_{2}\right\},\left\{b_{1}, b_{2}\right\}\right) Z_{p}\left(\left\{-a_{1},-a_{2}\right\},\left\{-b_{1},-b_{2}\right\}\right)}{Z_{p}\left(\left\{a_{1}, a_{2}\right\},\left\{-a_{1},-a_{2}\right\}\right) Z_{p}\left(\left\{b_{1}, b_{2}\right\},\left\{-b_{1},-b_{2}\right\}\right)} \\
& \times p^{-a_{1}-a_{2}-b_{1}-b_{2}}\left(1+\frac{z_{p}\left(1-a_{1}-b_{1}\right) z_{p}\left(1-a_{2}-b_{1}\right) z_{p}\left(b_{2}-b_{1}\right)}{z_{p}(1) z_{p}\left(-a_{1}-b_{1}\right) z_{p}\left(-a_{2}-b_{1}\right) z_{p}\left(1+b_{2}-b_{1}\right)}\right. \\
& \left.+\frac{z_{p}\left(1-a_{1}-b_{2}\right) z_{p}\left(1-a_{2}-b_{2}\right) z_{p}\left(b_{1}-b_{2}\right)}{z_{p}(1) z_{p}\left(-a_{1}-b_{2}\right) z_{p}\left(-a_{2}-b_{2}\right) z_{p}\left(1+b_{1}-b_{2}\right)}\right)
\end{aligned}
$$

and

$$
\begin{aligned}
W\left(a_{1}, b_{1} ; a_{2}, b_{2}\right)=P_{1}\left(a_{1}+b_{1}\right)\{ & P_{2}\left(a_{2}+b_{2}\right)-B_{3}\left(a_{1}, a_{2} ; b_{1}, b_{2}\right) \\
& \left.+P_{3}\left(a_{1}, b_{1}, b_{2}\right) P_{3}\left(b_{1}, a_{1}, a_{2}\right)\right\}
\end{aligned}
$$

with

$$
\begin{aligned}
& B_{3}\left(a_{1}, a_{2} ; b_{1}, b_{2}\right) \\
& =\sum_{p} \log ^{2} p\left(\frac{(p-1)^{2}\left(p^{a_{1}+b_{1}}-1\right)^{2} p^{a_{1}+b_{1}}}{\left(p^{a_{1}}-p^{a_{2}+1}\right)\left(-2 p^{a_{1}+b_{1}}+p^{a_{1}+b_{1}+1}+1\right)^{2}\left(p^{b_{1}}-p^{b_{2}+1}\right)}\right. \\
& \quad+\frac{C\left(a_{1}, a_{2} ; b_{1}, b_{2}\right)}{\left(p^{a_{1}}-p^{a_{2}+1}\right)\left(-2 p^{a_{1}+b_{1}}+p^{a_{1}+b_{1}+1}+1\right)\left(p^{b_{2}+1}-p^{b_{1}}\right)\left(p^{a_{2}+b_{2}+1}-1\right)} \\
& \left.\quad+\frac{1}{p^{a_{2}+b_{2}+1}-1}\right),
\end{aligned}
$$




$$
\begin{aligned}
& C\left(a_{1}, a_{2} ; b_{1}, b_{2}\right) \\
& \quad=-p^{a_{1}+b_{1}}+2 p^{a_{1}+b_{1}+1}-p^{a_{2}+b_{1}+2}-p^{2 a_{1}+2 b_{1}+1}+p^{a_{1}+a_{2}+2 b_{1}+1}-p^{a_{1}+b_{2}+2} \\
& \quad+p^{a_{2}+b_{2}+2}+p^{2 a_{1}+b_{1}+b_{2}+1}-2 p^{a_{1}+a_{2}+b_{1}+b_{2}+2}+p^{a_{1}+a_{2}+b_{1}+b_{2}+3},
\end{aligned}
$$

and

$$
\begin{aligned}
B_{4}\left(a_{1}, a_{2} ; b_{1}, b_{2}\right) \\
\quad=\sum_{p} \frac{\left(3-p^{1+a_{1}+b_{1}}-p^{1+a_{2}+b_{1}}-p^{1+a_{1}+b_{2}}-p^{1+a_{2}+b_{2}}+p^{2+a_{1}+a_{2}+b_{1}+b_{2}}\right) \log ^{4} p}{\left.\left(p^{1+a_{1}+b_{1}}-1\right)\left(p^{1+a_{2}+b_{1}}\right)-1\right)\left(p^{1+a_{1}+b_{2}}-1\right)\left(p^{1+a_{2}+b_{2}}-1\right)} .
\end{aligned}
$$

\subsection{Auxiliary functions}

For ease of reference we list the various auxiliary functions we have introduced in this example section.

$$
A(x)=\prod_{p} \frac{\left(1-1 / p^{1+x}\right)\left(1-2 / p+1 / p^{1+x}\right)}{(1-1 / p)^{2}}
$$

$A^{*}\left(a_{1}, a_{2}, b_{1}, b_{2}\right)=\prod_{p} \frac{Z_{p}\left(\left\{a_{1}, a_{2}\right\},\left\{b_{1}, b_{2}\right\}\right) Z_{p}\left(\left\{-a_{1},-a_{2}\right\},\left\{-b_{1},-b_{2}\right\}\right)}{Z_{p}\left(\left\{a_{1}, a_{2}\right\},\left\{-a_{1},-a_{2}\right\}\right) Z_{p}\left(\left\{b_{1}, b_{2}\right\},\left\{-b_{1},-b_{2}\right\}\right)}$

$$
\begin{aligned}
& \times p^{-a_{1}-a_{2}-b_{1}-b_{2}} \\
& \times\left(1+\frac{z_{p}\left(1-a_{1}-b_{1}\right) z_{p}\left(1-a_{2}-b_{1}\right) z_{p}\left(b_{2}-b_{1}\right)}{z_{p}(1) z_{p}\left(-a_{1}-b_{1}\right) z_{p}\left(-a_{2}-b_{1}\right) z_{p}\left(1+b_{2}-b_{1}\right)}\right.
\end{aligned}
$$

$$
\left.+\frac{z_{p}\left(1-a_{1}-b_{2}\right) z_{p}\left(1-a_{2}-b_{2}\right) z_{p}\left(b_{1}-b_{2}\right)}{z_{p}(1) z_{p}\left(-a_{1}-b_{2}\right) z_{p}\left(-a_{2}-b_{2}\right) z_{p}\left(1+b_{1}-b_{2}\right)}\right)
$$

$$
B(x)=\sum_{p}\left(\frac{\log p}{p^{1+x}-1}\right)^{2}
$$

$$
B_{2}\left(a, b_{1} ; b_{2}, b_{3}\right)=\sum_{p} \frac{(p-1) p^{2 b_{1}}\left(p^{a+b_{1}}-1\right)\left(p^{a+b_{1}}-p\right) \log ^{2} p}{\left(-2 p^{a+b_{1}}+p^{a+b_{1}+1}+1\right)^{2}\left(p^{b_{1}}-p^{b_{2}+1}\right)\left(p^{b_{1}}-p^{b_{3}+1}\right)}
$$




$$
\begin{aligned}
& B_{3}\left(a_{1}, a_{2} ; b_{1}, b_{2}\right)=\sum_{p} \log ^{2} p\left(\frac{(p-1)^{2}\left(p^{a_{1}+b_{1}}-1\right)^{2} p^{a_{1}+b_{1}}}{\left(p^{a_{1}}-p^{a_{2}+1}\right)\left(-2 p^{a_{1}+b_{1}}+p^{a_{1}+b_{1}+1}+1\right)^{2}}\right. \\
& +\frac{C\left(a_{1}, a_{2} ; b_{1}, b_{2}\right)}{\left(p^{a_{1}}-p^{a_{2}+1}\right)\left(-2 p^{a_{1}+b_{1}}+p^{a_{1}+b_{1}+1}+1\right)\left(p^{b_{2}+1}-p^{b_{1}}\right)} \\
& \left(p^{a_{2}+b_{2}+1}-1\right) \\
& \left.+\frac{1}{p^{a_{2}+b_{2}+1}-1}\right) \\
& \left(3-p^{1+a_{1}+b_{1}}-p^{1+a_{2}+b_{1}}-p^{1+a_{1}+b_{2}}-p^{1+a_{2}+b_{2}}\right. \\
& B_{4}\left(a_{1}, a_{2} ; b_{1}, b_{2}\right)=\sum_{p} \frac{\left.+p^{2+a_{1}+a_{2}+b_{1}+b_{2}}\right) \log ^{4} p}{\left(p^{1+a_{1}+b_{1}}-1\right)\left(p^{1+a_{2}+b_{1}}-1\right)\left(p^{1+a_{1}+b_{2}}-1\right)} \\
& \left(p^{1+a_{2}+b_{2}}-1\right) \\
& C\left(a_{1}, a_{2} ; b_{1}, b_{2}\right)=-p^{a_{1}+b_{1}}+2 p^{a_{1}+b_{1}+1}-p^{a_{2}+b_{1}+2}-p^{2 a_{1}+2 b_{1}+1} \\
& +p^{a_{1}+a_{2}+2 b_{1}+1}-p^{a_{1}+b_{2}+2}+p^{a_{2}+b_{2}+2}+p^{2 a_{1}+b_{1}+b_{2}+1} \\
& -2 p^{a_{1}+a_{2}+b_{1}+b_{2}+2}+p^{a_{1}+a_{2}+b_{1}+b_{2}+3} \\
& P_{1}(x)=e^{-\ell x} A(x) \zeta(1+x) \zeta(1-x) \\
& P_{2}(x)=\left(\frac{\zeta^{\prime}}{\zeta}\right)^{\prime}(1+x)-B(x) \\
& P_{3}(a, b, c)=B_{1}(a+b, a+c)+\frac{\zeta^{\prime}}{\zeta}(1+a+c)-\frac{\zeta^{\prime}}{\zeta}(1+c-b) \\
& Q(x, y)=-\sum_{p} \frac{\log ^{3} p}{p^{2+x+y}\left(1-1 / p^{1+x}\right)\left(1-1 / p^{1+y}\right)} \\
& W\left(a_{1}, b_{1} ; a_{2}, b_{2}\right)=P_{1}\left(a_{1}+b_{1}\right)\left\{P_{2}\left(a_{2}+b_{2}\right)-B_{3}\left(a_{1}, a_{2} ; b_{1}, b_{2}\right)\right. \\
& \left.+P_{3}\left(a_{1}, b_{1}, b_{2}\right) P_{3}\left(b_{1}, a_{1}, a_{2}\right)\right\} \\
& W_{1}\left(a, b_{1} ; b_{2}, b_{3}\right)=P_{1}\left(a+b_{1}\right)\left(P_{3}\left(a, b_{1}, b_{2}\right) P_{3}\left(a, b_{1}, b_{3}\right)-B_{2}\left(a, b_{1} ; b_{2}, b_{3}\right)\right)
\end{aligned}
$$

\section{References}

[1] E.B. Bogomolny and J.P. Keating, Gutzwiller's trace formula and spectral statistics: beyond the diagonal approximation, Phys. Rev. Lett. $77(8)$ (1996), 1472-1475. 
[2] — Random matrix theory and the Riemann zeros II:n-point correlations, Nonlinearity 9 (1996), 911-935.

[3] D. Bump and A. Gamburd, On the averages of characteristic polynomials from classical groups, Commun. Math. Phys. 265(1) (2006), 227-274; arXiv:math-ph/0502043.

[4] J.B. Conrey, Notes on eigenvalue distributions for the classical compact groups, in Recent Perspectives on Random Matrix Theory and Number Theory, LMS Lecture Note Series, 322, Cambridge University Press, Cambridge, 2005, 111-45.

[5] J.B. Conrey, D.W. Farmer, J.P. Keating, M.O. Rubinstein and N.C. Snaith, Integral moments of L-functions, Proc. Lond. Math. Soc. 91(1) (2005), 33-104; arXiv:math.nt/0206018.

[6] J.B. Conrey, D.W. Farmer and M.R. Zirnbauer, Autocorrelation of ratios of L-functions, Preprint, arXiv:0711.0718.

[7] - Howe pairs, supersymmetry, and ratios of random characteristic polynomials for the unitary groups $U(N)$, Preprint, arXiv:math$\mathrm{ph} / 0511024$.

[8] J.B. Conrey, P.J. Forrester and N.C. Snaith, Averages of ratios of characteristic polynomials for the compact classical groups, Int. Math. Res. Not. 7 (2005), 397-431.

[9] J.B. Conrey and N.C. Snaith, Triple correlation of the Riemann zeros, to be published in JNTB, arXiv:math/0610495.

[10] - Applications of the L-functions ratios conjectures, Proc. Lon. Math. Soc. 94(3) (2007), 594-646; arXiv:math.NT/0509480.

[11] H. Davenport, Multiplicative number theory, 3rd edn, Springer-Verlag, New York, 2000.

[12] F.J. Dyson, Statistical theory of the energy levels of complex systems, i, ii and iii, J. Math. Phys. 3 (1962), 140-175.

[13] H.M. Edwards, Riemann's zeta function, Dover Publications, Inc.

[14] P. Gao, n-level density of the low-lying zeros of quadratic Dirichlet L-functions, $\mathrm{PhD}$ thesis, University of Michigan, 2005.

[15] C.P. Hughes and S.J. Miller, Low-lying zeros of L-functions with orthogonal symmetry, Duke Math. J. 136(1) (2007), 115-172. 
[16] S.G. Krantz, Function theory of several complex variables, 2nd edn, American Mathematical Society, Providence, Rhode Island, 1992.

[17] H.L. Montgomery, The pair correlation of the zeta function, Proc. Symp. Pure Math. 24 (1973), 181-93.

[18] A.M. Odlyzko, The $10^{20}$ th zero of the Riemann zeta function and 70 million of its neighbors, Preprint, 1989, http://www.dtc.umn.edu/ odlyzko/unpublished/index.html.

[19] M.O. Rubinstein, Low-lying zeros of L-functions and random matrix theory, Duke Math. J. 109(1) (2001), 147-181.

[20] Z. Rudnick and P. Sarnak, Zeros of principal L-functions and random matrix theory, Duke Math. J. 81(2) (1996), 269-322.

[21] E.C. Titchmarsh, The theory of the Riemann zeta function, 2nd edn, Clarendon Press, Oxford, 1986.

American Institute of Mathematics

360 Portage Aveneu

Palo Alto, CA

94306-2244

U.S.A.

E-mail address: conrey@aimath.org

School of Mathematics

UNIVERSITY OF BRISTOL

UNIVERSITY WALK

BRISTO, BS8 1TW

United Kingdom

E-mail address: n.c.snaith@bristol.ac.uk

Received March 19, 2008 